ARTICLE

\title{
TcpC inhibits neutrophil extracellular trap formation by enhancing ubiquitination mediated degradation of peptidylarginine deiminase 4
}


Meng-qing Qian',3, Da-yong Zhang ${ }^{1,3}$, Jun-ping Guo 1,3, Wei Gao1,3, Na-ru Zhang ${ }^{1,3}$ \& Jian-ping Pan (1) 1,3凶

$\mathrm{TcpC}$ is a multifunctional virulence factor of uropathogenic E. coli (UPEC). Neutrophil extracellular trap formation (NETosis) is a crucial anti-infection mechanism of neutrophils. Here we show the influence of TcpC on NETosis and related mechanisms. We show NETosis in the context of a pyelonephritis mouse model induced by $\mathrm{TcpC}$-secreting wild-type $E$. coli CFT073 (CFT073 ${ }^{\text {wt }}$ ) and LPS-induced in vitro NETosis with CFT073 ${ }^{\text {wt }}$ or recombinant TcpC $(r T c p C)$-treated neutrophils are inhibited. $\mathrm{rTcpC}$ enters neutrophils through caveolinmediated endocytosis and inhibits LPS-induced production of ROS, proinflammatory cytokines and protein but not mRNA levels of peptidylarginine deiminase 4 (PAD4). rTcpC treatment enhances PAD4 ubiquitination and accumulation in proteasomes. Moreover, in vitro ubiquitination kit analyses show that $\mathrm{T} c \mathrm{C} C$ is a PAD4-targetd $\mathrm{E} 3$ ubiquitin-ligase. These data suggest that $\mathrm{T} \mathrm{CPC}$ inhibits NETosis primarily by serving as an E3 ligase that promotes degradation of PAD4. Our findings provide a novel mechanism underlying TcpCmediated innate immune evasion.

\footnotetext{
${ }^{1}$ Institute of Translational Medicine, Zhejiang University City College, Hangzhou, P. R. China. ${ }^{2}$ Department of Basic Medical Sciences, Zhejiang University School of Medicine, Hangzhou, P. R. China. ${ }^{3}$ Department of Clinical Medicine, Zhejiang University City College School of Medicine, Hangzhou, P. R. China.

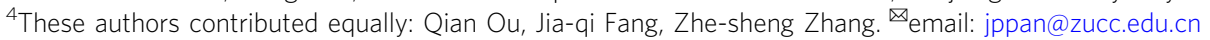


rinary tract infection (UTI) is one of the most common bacterial infections affecting 150 million people worldwide each year ${ }^{1,2}$. It is estimated that the medical burden of UTI exceeds $\$ 6$ billion annually ${ }^{3}$. These significant infections can be a serious health problem to reduce the quality of life. E. coli is the most common pathogen of UTIs, and $\sim 80 \%$ of UTI are caused by E. coli ${ }^{3}$. Urinary pathogenic E. coli (UPEC) can specifically adhere to and implant into urothelial mucosal epithelial cells, leading to UTIs, including asymptomatic bacteriuria, urethritis, urocystitis, and pyelonephritis $(\mathrm{PN})^{4}$.

It was found that most strains of UPEC secrete a protein containing the Toll/interleukin 1 receptor (TIR) domain or $\mathrm{TcpC}^{5}$. TcpC is an important virulence factor of UPEC that subverts the toll like receptor (TLR) signaling pathway by direct association of myeloid differentiation factor 88 (MyD88) ${ }^{5,6}$. TcpC inhibits the bactericidal activity of macrophages and promotes the survival of UPEC in the host, which favors the pathogenicity of the pathogen ${ }^{5,7}$.

The characteristic pathological changes of acute pyelonephritis are neutrophil infiltration ${ }^{5}$. It is well-known that neutrophils are important effector arms of the innate immune system. Neutrophils play crucial roles in clearance of extracellular bacterial infection by phagocytosis. Over the past years, increasing evidence has demonstrated that neutrophils have, in addition to phagocytosis, a special anti-infection mechanism that produces neutrophil extracellular traps (NETs), the process of NETs formation is referred to as NETosis ${ }^{8}$. NETs are three dimensional meshwork consisting of chromatin, antimicrobial components such as myeloperoxidase (MPO) and neutrophil elastase (NE) ${ }^{9}$. These fibrous networks can catch and eliminate bacteria, fungi, and viral particles as well ${ }^{9-11}$. NETosis can be induced by a wide range of substances, including bacteria, lipopolyssacharide (LPS), phorbol-12-myristate-13-acetate (PMA), and viruses, etc ${ }^{12}$. The specific requirements for NETs formation depend on the stimulus, but histone citrullination mediated by the peptidylarginine deiminase 4 (PAD4) has been demonstrated to be an essential step in NETosis $9,13-15$

PAD4 is primarily expressed in neutrophils, it catalyzes the conversion of histone arginine to citrullination ${ }^{16}$. Citrullination is a post-translational modification that plays an important role in many physiological processes such as cell differentiation and renewing, but abnormal citrullination, however, can lead to the development of diseases, including autoimmune diseases, cancers, and cardiovascular diseases ${ }^{13}$. Numerous stimuli have been confirmed to activate PAD4 and NETosis ${ }^{17,18}$.

There are reactive oxygen species (ROS)-dependent and ROSindependent NETosis. ROS-dependent NETs release requires the production of ROS by NADPH oxidase ${ }^{19}$. ROS stimulate MPO to trigger the activation and translocation of NE from the azurophilic granules to the nucleus where NEs digest nucleosomal histones and promote chromatin relaxation, which favors the citrullination of histone $(\mathrm{CitH} 3)$ by $\mathrm{PAD} 4{ }^{20}$. Citrullination of histone leads to chromatin decondensation, which causes entropic chromatin swelling 9 . Subsequently, MPO associate with chromatin and, synergistically with $\mathrm{NE}$ and PAD4, promote massive chromatin decondensation ${ }^{15}$. In association with diverse granular and cytoplasmic proteins, decondensed chromatin is eventually released into the extracellular space ${ }^{17}$.

Although TcpC was demonstrated to play a crucial role in the immune evasion of macrophage-mediated innate immunity $5,6,21$, its influence on NETosis remains elusive. In the present study, we show that TcpC suppresses NETosis by promoting degradation of PAD4 through ubiquitin proteasome pathway. Our findings provide not only a novel mechanism underlying TcpC-mediated innate immune evasion, but also new insight into the pathogenicity of pathological microbes.

\section{Results}

NETosis is inhibited in PN mouse model induced by TcpCsecreting wild-type CFT073 (CFT073wt) and in CFT073 ${ }^{\text {wt }}$. treated neutrophils. The PN mouse models were induced by urethral instillation of CFT073 ${ }^{\text {wt }}$ or tcpc-knock out CFT073 mutant (CFT073 ${ }^{\Delta t c p c}$ ) as described previously ${ }^{5,21,22}$. Abscesses in kidneys could be observed in CFT073 ${ }^{\mathrm{wt}}$-induced PN mouse model, no abscess was present in kidneys from CFT073 ${ }^{\Delta t c p c_{-}}$ infected mice (Fig. 1a, b). Accordingly, significantly increased infiltrates of neutrophil were observed in kidneys from the CFT073 $3^{\text {th }}$ group compared with those in kidneys from the CFT073 ${ }^{\Delta t c p c}$ group (Fig. 1c). These data demonstrate again that TcpC is a crucial virulence factor of UPEC. In situ NETosis in kidneys from PN mouse model induced by CFT073 ${ }^{\mathrm{wt}}$ was significantly inhibited when compared with that in kidneys from the CFT073 ${ }^{\Delta t c p c}$ infected group (Fig. 1d, e). To further confirm that this inhibition of in situ NETosis was caused by CFT073wt, human neutrophils were separately co-cultured in transwell with CFT073 ${ }^{\mathrm{wt}}$ or CFT073 ${ }^{\Delta t c p c}$ at a multiplicity of infection $(\mathrm{MOI})=$ 100 for $12 \mathrm{~h} .4 \mathrm{~h}$ before the end of the co-culture, $1 \mu \mathrm{g} / \mathrm{ml}$ LPS was added into the corresponding wells and NETosis was examined. NETosis in the CFT073 ${ }^{\mathrm{wt}}+$ LPS group was significantly inhibited compared with the LPS or CFT073 ${ }^{\Delta t c p c}+$ LPS groups. No significant inhibition of NETosis was observed in the group of CFT073 ${ }^{\Delta t c p c}+$ LPS compared with the LPS treatment group (Fig. 1f, g). These data demonstrate that CFT073wt inhibits NETosis.

Recombinant TcpC protein inhibits NETosis. Since CFT073 treatment caused inhibition of NETosis, recombinant TcpC protein (rTcpC) was prepared and its influence on NETosis was examined. The entry of $\mathrm{rTcpC}$ into neutrophils was verified by confocal and western blot analyses. rTcpC could enter the cells gradually with the treatment time (Supplementary Fig. 1a-c). Endocytosis is the process by which cells actively internalize molecules and surface proteins via an endocytic vesicle ${ }^{23}$. In order to confirm which endocytic vesicle mediated the entry of rTcpC, the cells were treated by clathrin-mediated endocytosis inhibitor dynasore $^{24,25}$ and caveolin-dependent endocytosis inhibitor Methyl- $\beta$-cyclodextrin $(\mathrm{MCD})^{24,26}$ for 30 min before treatment with $\mathrm{rTcpC}$ respectively. MCD but not dynasore blocked the entry of rTcpC into neutrophils, demonstrating that $\mathrm{rTcpC}$ could get into neutrophils through caveolin-mediated endocytosis (Supplementary Fig. 1d-f). LPS and PMA-induced ROS-dependent NETs formation, which is in line with previous report ${ }^{27}$. Although TAK-242, a TLR4 inhibitor ${ }^{28}$, did not influence PMAinduced NETosis, it completely blocked the LPS-induced NETosis. In the presence of $\mathrm{rTcpC}$, both the LPS- and PMAinduced NETosis were significantly suppressed (Fig. 2a-c). In ROS-dependent NETosis, oxidative stress occurs in neutrophils. NADPH oxidase catalyzes the intracellular $\mathrm{O}_{2}$ to generate ROS that destroy pathogenic bacteria and promote translocation of MPO and NE to the nucleus, where NE proteolytically processes histones to disrupt chromatin packaging and MPO binds decondensing chromatin ${ }^{29}$, meanwhile expression of IL-1 $\beta$, IL-6, TNF- $\alpha$, and other proinflammatory factors is enhanced to achieve antibacterial effect ${ }^{30,31}$. The influence of $\mathrm{rTcpC}$ on ROS and proinflammatory cytokine production was examined. In the presence of rTcpC, LPS-induced production of ROS was profoundly inhibited while $\mathrm{rTcpC}$ itself did not affect the generation of ROS (Fig. 2d-f). Upon stimulation of LPS, enhanced expression of IL- $1 \beta$, IL- 6 , and TNF- $\alpha$ occurred in neutrophils. However, rTcpC substantially inhibited this LPS-induced production of proinflammatory cytokines at both protein and mRNA levels (Fig. 2g, h). Moreover, when neutrophils were treated by 
a
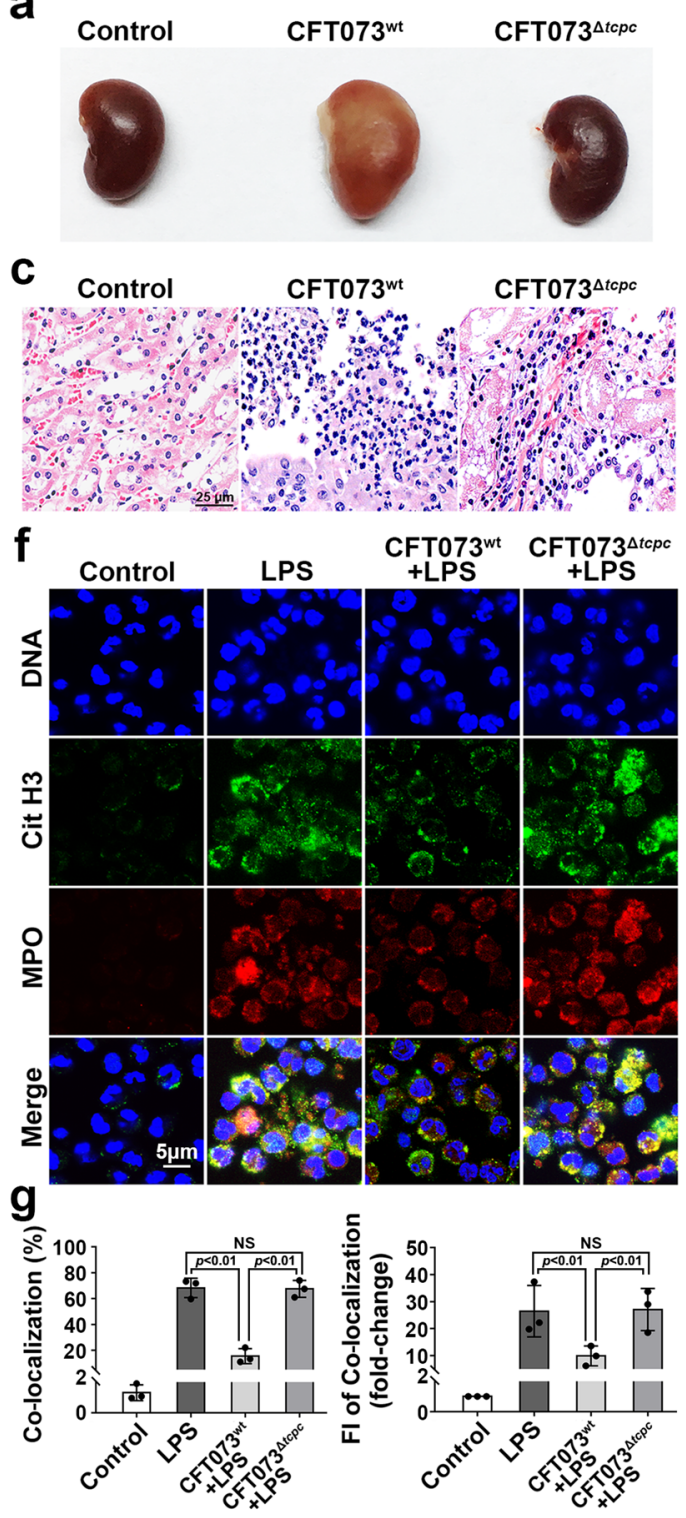

b

\begin{tabular}{|c|c|c|}
\hline Mouse strain & Group & Abscess formation (\%) \\
\hline \multirow{3}{*}{ C57BL/6 } & Control & $0 / 5(0)$ \\
\cline { 2 - 3 } & CFT073 $^{\text {wt }}$ & $4 / 5(80)$ \\
\cline { 2 - 3 } & CFT073 $^{\text {ttcpc }}$ & $0 / 5(0)$ \\
\hline
\end{tabular}

d
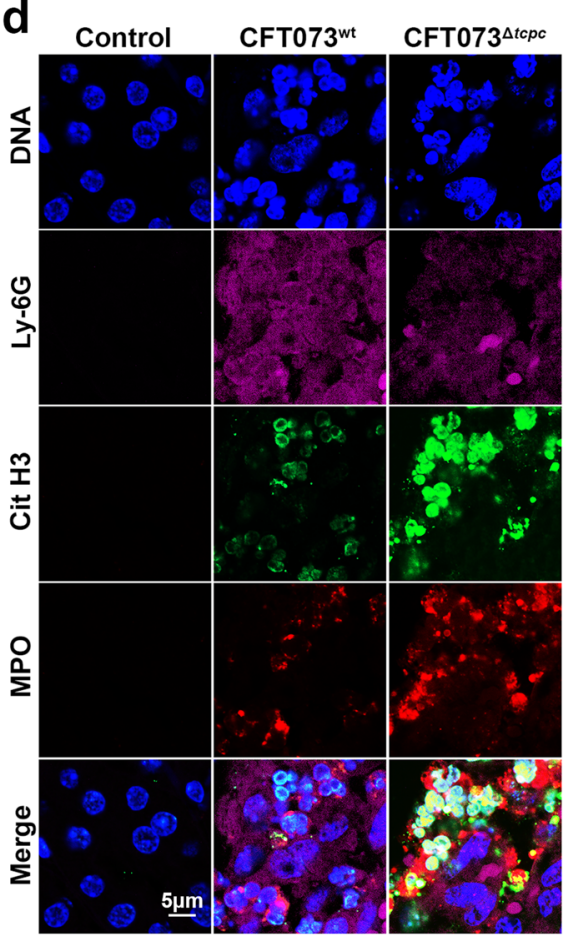

$\mathbf{e}_{\overline{0}}$

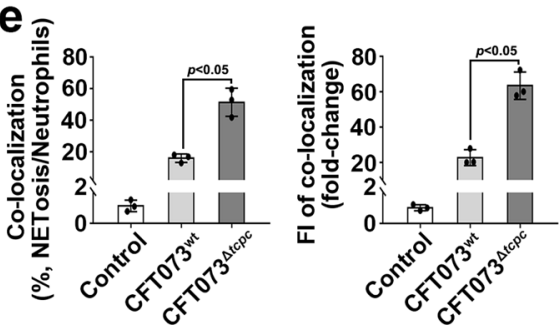

Fig. 1 NETosis is inhibited in Kidneys of CFT073wt induced pyelonephritis murine model and in CFT073wt-treated neutrophils. a Gross pathological

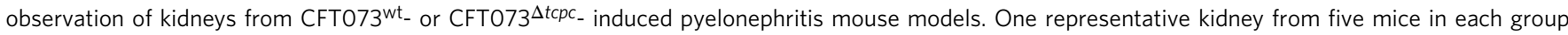
was shown. $n=5$. $\mathbf{b}$ Abscess formation of kidneys in mice with different treatment. $\mathbf{c}$ Histological examination of kidneys from CFT073wt- or CFT073 ${ }^{\Delta t c p c}$ induced pyelonephritis mouse models. Scale bar $=25 \mu \mathrm{m}$. One representative image of kidneys from five mice in each group was shown, $n=3$. $\mathbf{d}$ Confocal

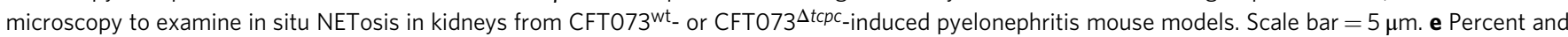
$\mathrm{FI}$ of co-localization reflecting the NETosis levels in experiments as described in $\mathbf{d}$. Mean \pm SD of data from five kidneys in each group were shown, $n=5$. FI in the control group was set as 1.0, $p<0.05$ and $p<0.01$ were considered to be statistically significant and extremely significant respectively. NS not significant. $\mathbf{f}$ Examination of in vitro LPS-induced NETosis of CFT073wt or CFT073 ${ }^{\Delta t c p c}$ treated neutrophils by confocal microscopy. Scale bar $=5 \mu \mathrm{m}$. Images are representative of neutrophils from three different donors, $n=3$. $\mathbf{g}$ Percent and FI of co-localization reflecting the NETosis levels in experiments as described in $\mathbf{f}$. Mean \pm SD of three independent experiments were shown, $n=3 . p<0.05$ and $p<0.01$ were considered to be statistically significant and extremely significant respectively. NS not significant. $p$-values were derived by Dunnett and Mann-Whitney tests. Source data for panel $\mathbf{e}, \mathbf{g}$ are provided in the separate Source Data file.

CFT073 ${ }^{\mathrm{wt}}$, results with same trend were also obtained (Supplementary Fig. 2a, b). These data suggest that TcpC inhibits ROSdependent NETosis.

rTcpc not only inhibits the citrullination of chromatin histone, but also affects the transcription of related genes in nucleus. During the ROS-dependent NETosis, NE digested histones are citrullinated by PAD4, resulting in chromatin decondensation ${ }^{17,32}$. Dynamic analyses of the influence of $\mathrm{rTcpC}$ on chromatin decondensation were carried out as described in previous reports ${ }^{33,34}$. Decondensation of chromatin increased along with the time in the LPS group. In the presence of rTcpC, however, the LPSinduced chromatin decondensation was significantly inhibited (Fig. 3a-c). Since CitH3 is also one of the indicators of chromatin decondensation ${ }^{35}$, CitH3 levels were dynamically analyzed. As shown in Fig. 3d, e, CitH3 levels also increased along with the time in LPS group, but this LPS-induced increase of CitH3 was suppressed significantly in the LPS $+\mathrm{rTcpC}$ group. Furthermore, rTcpC itself dynamically suppressed the levels of CitH3. Taken 


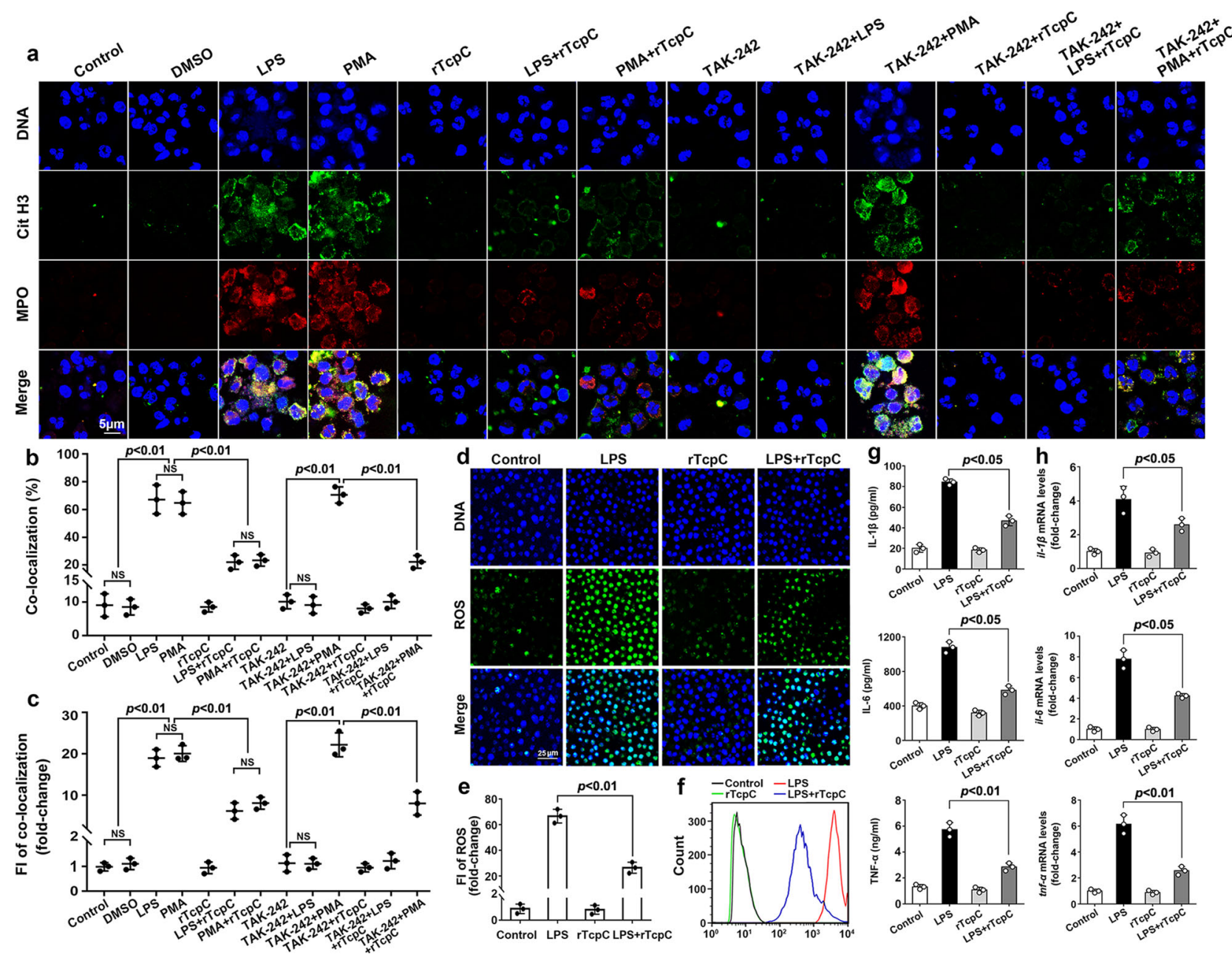

Fig. 2 rTcpC inhibits NETosis. a Confocal microscopy to detect the influence of $r$ TcpC on ROS-dependent NETosis. Scale bar $=5 \mu \mathrm{m}$. $\mathbf{b}$ Percent of colocalization reflecting the NETosis levels in experiments as described in $\mathbf{a}$. Mean \pm SD of three independent experiments were shown. $\mathbf{c}$ Fl of co-localization in experiments as described in $\mathbf{a} . \mathbf{b}, \mathbf{c} p<0.01$ was considered to be extremely significant. The FI reflecting NETosis level in the control group was set as 1.0 . Mean \pm SD of three independent experiments were shown. $p<0.01$ was considered to be extremely significant. NS not significant. $p$-values were derived by Mann-Whitney multiple comparisons test. $\mathbf{d}$ Confocal analyses of the influence of $r$ TcpC on ROS production. Scale bar $=25 \mu \mathrm{m}$. Images shown are representative of three independent experiments. $n=3$. e FI of ROS were analyzed by ImageJ software. Mean \pm SD of three independent experiments were shown. $p<0.01$ was considered to be extremely significant. $p$-values were derived by Dunnett comparison test. $\mathbf{f}$ Flow cytometry detection of the effects of rTcpC on ROS production in LPS-induced NETosis. $\mathbf{g}$, $\mathbf{h}$ Protein and mRNA levels of IL-1 $\beta$, IL- 6 , TNF- $\alpha$ were detected by ELISA and qRT-PCR, respectively. Mean \pm SD of three independent experiments $(n=3) . p<0.05$ and $p<0.01$ were considered to be statistically significant and extremely significant respectively. $p$-values were derived by Dunnett comparison test. Source data for panel $\mathbf{b}, \mathbf{c}, \mathbf{e}, \mathbf{g}, \mathbf{h}$ are provided in the separate Source Data file.

together, these data further indicate that $\mathrm{rTcpC}$ can inhibit chromatin depolymerization to form NETs. To determine the relevance of transcriptional activity during NETosis, a genome-wide transcriptomics analysis of neutrophils treated with LPS and/or rTcpC was conducted. $\mathrm{rTcpC}$ promoted gene transcripts down-regulation more than up-regulation (Fig. 4a). A large number of gene transcripts related to superoxidation and cytokines of ROS-dependent NETosis were inhibited (Fig. 4b), but ubiquitination-related gene transcripts were significantly up-regulated in rTcpC-treated neutrophils (Fig. 4c). Therefore, TcpC not only significantly inhibits the chromatin depolymerization, but also affects gene transcription during NETosis.

rTcpC dose-dependently and dynamically decreases protein but not mRNA levels of PAD4 in neutrophils. Because PAD4mediated histone citrullination is the essential step in NETs formation $9,13,36$, dose-dependent and dynamic analyses of the influence of $\mathrm{rTcpC}$ on PAD4 expression were examined. In accordance with the results of transcriptomics analyses (Fig. 4b), upon LPS stimulation, both mRNA and protein levels of PAD4 increased significantly (Fig. 5a-f). Although rTcpC treatment did not affect LPS-induced mRNA expression of PAD4 (Figs. 4b and $5 \mathrm{a}, \mathrm{d}), \mathrm{rTcpC}$ itself could dose-dependently inhibit protein levels of PAD4 compared with the control group (Fig. 5b, c). Importantly, dose-dependent and dynamical inhibition of PAD4 protein levels were observed in the LPS $+\mathrm{rTcpC}$ group when compared with the LPS group (Fig. 5b, $c$ and e, f). Furthermore, when neutrophils were treated with CFT073 ${ }^{\text {wt }}$ and CFT073 ${ }^{\Delta t c p c}$, similar results were obtained (Supplementary Fig. 3a, b) These data show that TcpC decreases protein but not mRNA levels of PAD4 in neutrophils.

rTcpC is an E3 ubiquitin ligase that promotes ubiquitination of PAD4. Ubiquitination is an important protein post-translational modification pathway in eukaryotic cells ${ }^{37}$. Ubiquitination is mediated by ubiquitin-activating (E1), ubiquitin-conjugating (E2), 



Fig. 3 rTcpc inhibits chromatin decondensation in NETosis. a Dynamic analyses of chromatin decondensation by Sytoxgreen staining. Scale bar $=5 \mu$ m. b Image J analyses of DNA fluorescence area from experiments as described in $\mathbf{a}$. $\mathbf{a}$, $\mathbf{b}$ Mean \pm SD are derived from three independent experiments in which five independent areas per condition were measured. $p<0.05$ compared with LPS group, $p$-values were derived by Dunnett test. $\mathbf{c}$ Image J analyses of confocal images from experiments as described in a. Mean \pm SD of three independent experiments were shown. $p<0.05$ and $p<0.01$ were considered to be statistically significant and extremely significant respectively, $p$-values were derived by Dunnett and Mann-Whitney tests. $\mathbf{d}$ Dynamic analyses of CitH3 in neutrophils with different treatment by western blot. e Gray scale analyses of bands from experiment as described in $\mathbf{d}$. Mean \pm SD of three independent experiments were shown, $n=3 . p<0.05$ and $p<0.01$ were considered to be statistically significant and extremely significant respectively, $p$-values were derived by Dunnett and Mann-Whitney tests. All western blots in panels $\mathbf{d}$ are provided as uncropped blots in Gels and Blots of Source Data file. Source data for panel b, c, e are provided in the separate Source Data file.

and ubiquitin-ligating (E3) enzyme cascade reactions ${ }^{38}$. In the process of ubiquitination, E3 binds not only to E2, but also specifically to substrate proteins ${ }^{39}$. In human genome, $>600$ annotated E3 ligases have been found, they are divided into three types of domains: RING, HETC, and $\mathrm{RBR}^{40}$. In the process of ubiquitination by HETC E3 ligase, the conserved cysteine (Cys) residue at Nterminus of the enzyme is necessary ${ }^{37,41}$. HECT E3 ligase contains tryptophan-tryptophan (WW) domains that bind to prolinetyrosine $(\mathrm{P}-\mathrm{Y})$ motif containing substrates ${ }^{42-44}$. In our previous studies, we have demonstrated for the first time that TcpC is a HECT family E3 ubiquitin ligase that can promote ubiquitination of

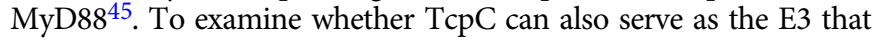
promote PAD4 ubiquitination, we did bioinformatics analyses at first and found that human and mouse PAD4 contain P-Y motifs (Fig. 6a, b), suggesting that PAD4 is probably one of the TcpC E3 enzyme substrates. Then, the influence of rTcpC on ubiquitination of PAD4 was examined by co-immunoprecipitation and immunoblotting assays. Although $\mathrm{rTcpC}$ treatment decreased the PAD4 

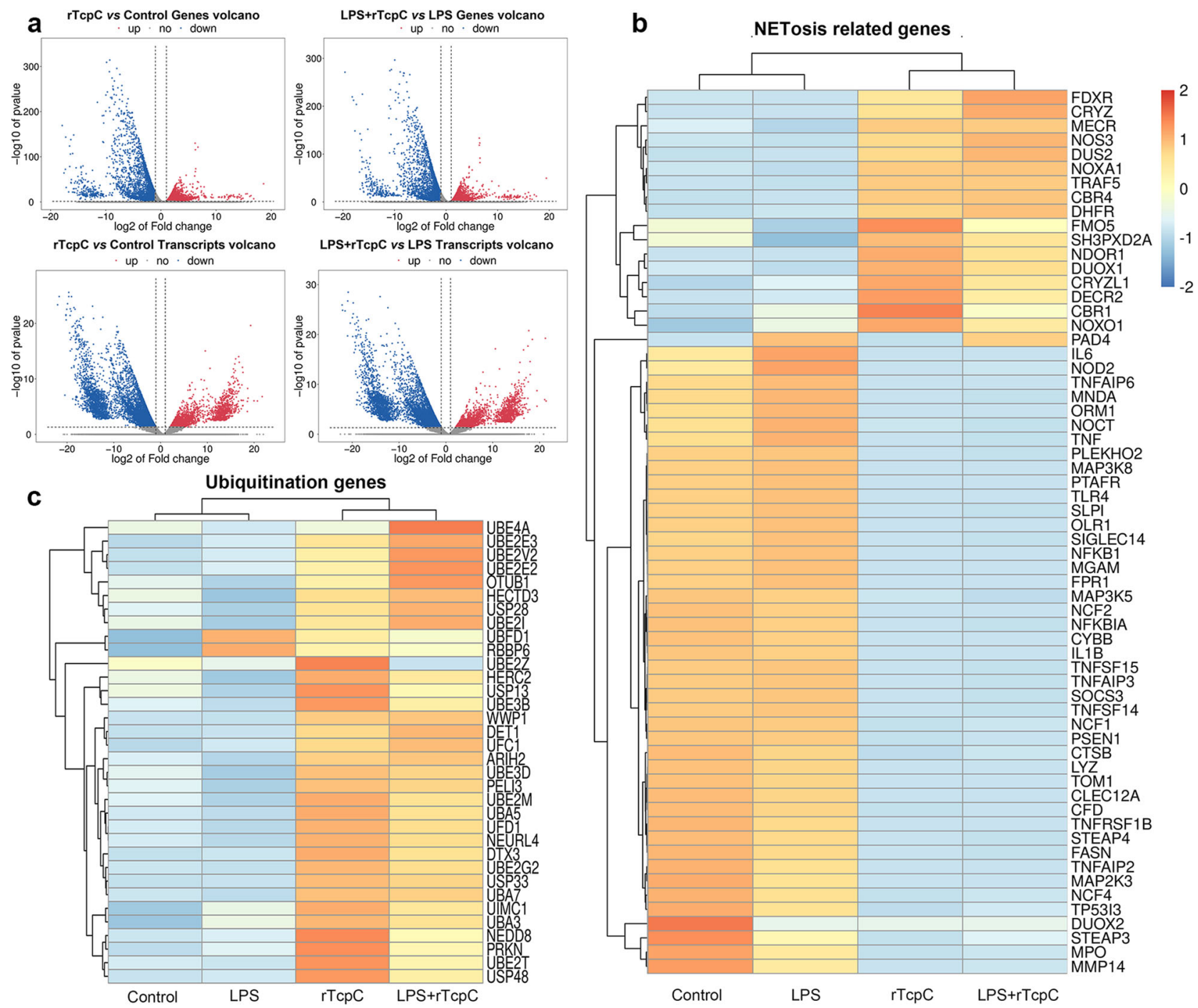

C

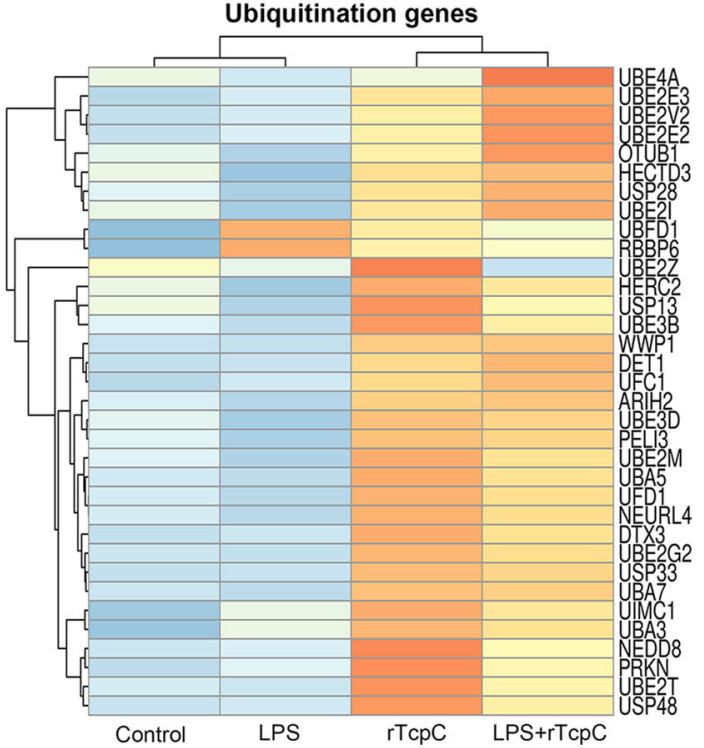

Control

LPS

Fig. 4 rTcpC affects the transcription of genes in neutrophils. a Genes and transcripts volcano in different treatment groups. b NETosis-related gene transcription levels in untreated (Control), LPS-, rTcpC-, or LPS + TcpC treated neutrophils. c Ubiquitination-related gene transcription levels in different treatment groups of neutrophils. a-c Data are representative of three biological repeats $(n=3)$. Source data for panel a-c are provided in the separate Source Data file.

protein level in lysates of neutrophils, the ubiquitination of PAD4 was significantly enhanced compared with the LPS-treated group. When the proteasome activity was blocked by MG-132, the ubiquitination of PAD4 was enhanced further in the rTcpC-treated groups (Fig. 6c, d). These data demonstrate that $\mathrm{rTcpC}$ promotes ubiquitination of PAD4. At last, the PAD4-targeted E3 activity of rTcpC was further examined by in vitro ubiquitination kit analyses which mimic the ubiquitination process as previously described $^{46,47}$. In these assays, the GST-MuRF (E3) and its substrate S5a were served as the positive control. When the E3 in the kit was replaced by $\mathrm{rTcpC}$ and lysates of neutrophils and commercially available human recombinant PAD4 (Hu-rPAD4) used as the substrates, substantially enhanced ubiquitination of PAD4 could be observed. Furthermore, this rTcpC mediated enhanced ubiquitination of PAD4 could be abrogated when the $\mathrm{E} 3$ inhibitor Nutlin-3 was employed (Fig. 6e). These data firmly demonstrate that TcpC is an E3 ubiquitin ligase which promotes ubiquitination of PAD4.

TcpC promotes accumulation of PAD4 in proteasomes. In the process of ubiquitination, E3 ubiquitin ligase transports substrate protein to proteasome for degradation ${ }^{48,49}$. In order to verify if
rTcpC treatment leads to accumulation of PAD4 in the proteasomes, confocal microscopy was employed to examine colocalization of PAD4 with the proteasome marker, non-ATPase 2 (PSMD2) 50,51 . Dynamic analyses of PAD4 accumulation in proteasomes showed that, in the absence of MG-132, colocalization of PAD4 and PSMD2 increased 60 min later, and reached a plateau $120 \mathrm{~min}$ after $\mathrm{rTcpC}$ treatment (Fig. $7 \mathrm{a}-\mathrm{c})$. In the presence of MG-132, however, co-localization of PAD4 with PSMD2 increased steadily after 60 min treatment with $\mathrm{rTcpC}$ (Fig. $7 \mathrm{~d}-\mathrm{f}$ ). These data suggest that $\mathrm{rTcpC}$ promotes PAD4 accumulation in proteasomes in LPS-stimulated neutrophils.

C12S point mutant $r T c p C-C 12 S$ loses the ability to inhibit NETosis. In our previous studies, we have demonstrated that $\mathrm{C} 12$ is the key amino acid to retain the $\mathrm{E} 3$ activity of $\mathrm{TcpC}^{45}$, the influence of C12S point mutant $\mathrm{rTcpC}-\mathrm{C} 12 \mathrm{~S}$ on degradation of PAD4 and LPS-induced NETosis was examined. Compared with the rTcpC prototype, rTcpC-C12S showed significantly attenuated activity to promote degradation of PAD4 (Fig. 8a, b) and to inhibit LPS-induced NETosis (Fig. 8c-e), suggesting further 

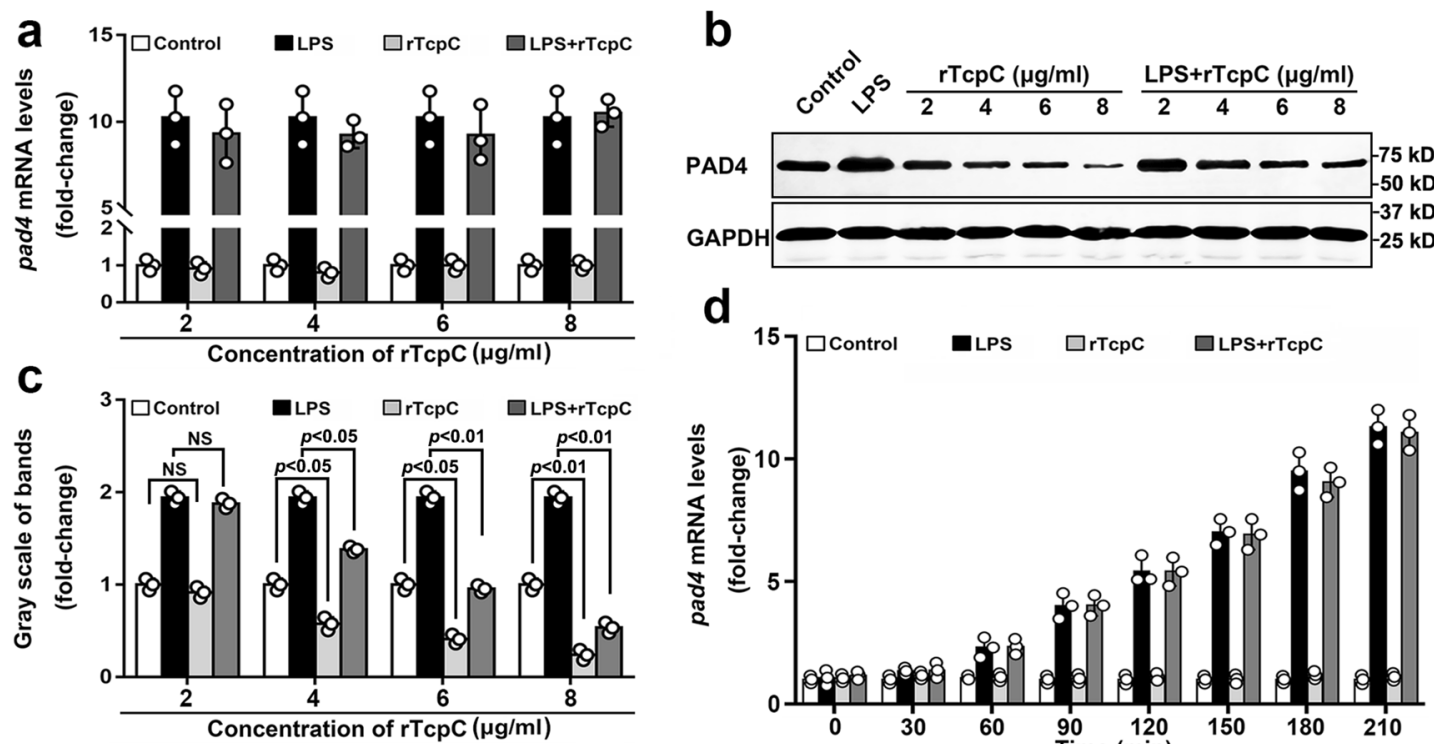

d
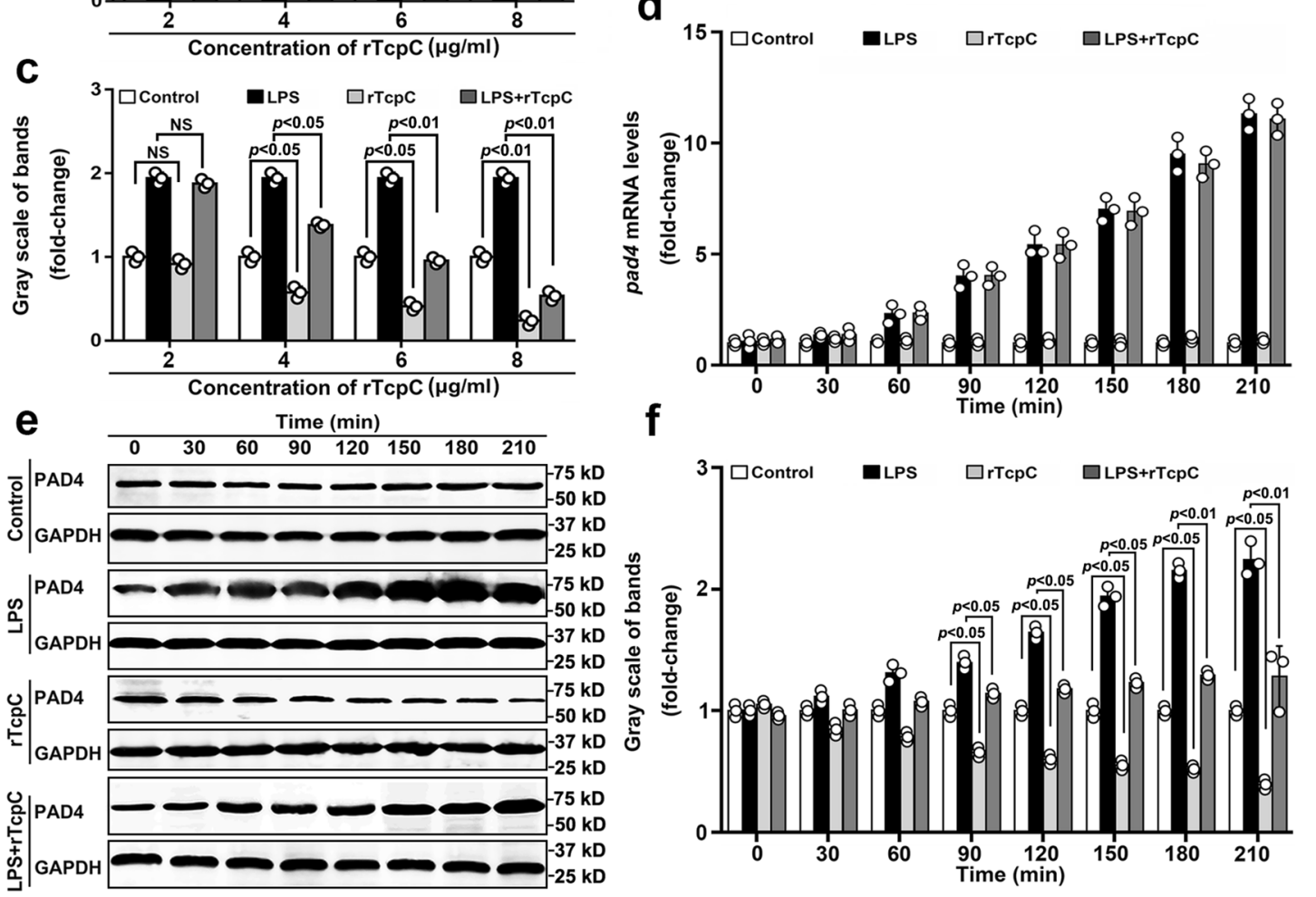

Fig. 5 rTcpC inhibits protein but not mRNA levels of PAD4 in LPS-treated neutrophils. a qRT-PCR to examine mRNA levels of PAD4 in different treatment groups of neutrophils. Data are from three independent experiments $(n=3)$ with three replicates per condition. $\mathbf{b}$ Western blot analyses of PAD4 protein levels in neutrophils with different treatment. c Gray scale analyses of bands from experiments as described in $\mathbf{b}$. Gray scale values reflecting the PAD4 levels in the control group were set as 1.0. Mean \pm SD of three independent experiments were shown. $p<0.05$ and $p<0.01$ were considered to be statistically significant and extremely significant respectively, NS not significant. d Dynamic analyses of the influence of $r T c p C$ on pad4 mRNA levels in neutrophils with different treatment. The data of pad4 mRNA levels are from 3 independent experiments $(n=3)$. e Dynamic analyses of PAD4 in neutrophils with different treatment by western blot. $\mathbf{f}$ Gray scale analyses of bands from experiment as described in $\mathbf{e}$. Mean \pm SD of three independent experiments were shown. $p$-values were derived by Dunnett and Mann-Whitney tests. All western blots in panels $\mathbf{b}$, e are provided as uncropped blots in Gels and Blots of Source Data file. Source data for panel $\mathbf{a}, \mathbf{c}, \mathbf{d}, \mathbf{f}$ are provided in the separate Source Data file.

that the inhibitory effect of TcpC on NETosis is mediated by promoting degradation of PAD4.

\section{Discussion}

Although the pathophysiological roles of NETosis have not been fully understood, NETosis is an important anti-infection mechanism of neutrophils. In ROS-dependent NETosis, NADPH oxidase complex catalyzes $\mathrm{O}_{2}$ to produce ROS which activate transcription and activation of downstream NETosis essential enzyme genes ${ }^{52}$. Among them, PAD4 is of the most important. The conversion of histone arginine into citrulline catalyzed by PAD4, which reduces the positive charges of histone and causes chromatin entropic swelling, is the most important event that drives the release of NETs ${ }^{9}$. After chromatin entropic swelling the process of NET formation cannot be reversed. Therefore, histone citrullination can be regarded as the "turningpoint" in NETosis. Although the exact signaling pathway that regulates PAD4 expression has not been clarified, manipulating PAD4 to inhibit NETosis might be a vital strategy for pathogens to evade effector function of neutrophils. On the one hand, it was found that TcpC impedes TLR signaling pathway, hereby inhibiting macrophage-mediated innate immunity ${ }^{5,21}$. On the other hand, TcpC promotes kidney cells to produce CXCL2 chemokine which attracts neutrophil infiltration in kidney, the characteristic pathological changes of acute $\mathrm{PN}^{22}$. But the influence of $\mathrm{TcpC}$ on NETosis has been remained elusive.

In the present study, we show that in situ NETosis in kidneys of PN murine model induced by CFT073 ${ }^{\text {wt }}$ was significantly inhibited compared with that in CFT073 ${ }^{\Delta t c p c}$ infected mouse (Fig. 1d, e). In line with the results of in situ NETosis, LPSinduced in vitro NETosis was also suppressed significantly in CFT073wt - (Fig. 1f, g) or rTcpC-treated (Fig. 2a-c) human neutrophils. These data demonstrate that TcpC suppresses NETosis.

During ROS-dependent NETosis, ROS is produced to kill pathogens and stimulate NE to digest histone for citrullination by PAD4 in nucleus ${ }^{20,53}$. Furthermore, proinflammatory cytokines such as IL-1 $\beta$, IL-6, TNF- $\alpha$ are up-regulated when neutrophils undergo NETosis ${ }^{54}$. In order to explore the mechanisms underlying TcpC-mediated inhibition of NETosis, we examined the 
a

P-Y motifs of human PAD4

MAQGTLIRVTPEQPTHAVCVLGTLTQLDICSSAPEDCTSFSINASPGVVV DIAHGPPAKKKSTGSSTWLDPGVEVTLTMKVASGSTGDQKVQISYYG KTPPVKALLYLTGVEISLCADITRTGKVKPTRAVKDQRTWTWGPCGQ GATLENNCDRDNLESSAMDCEDDEVLDSEDLQDMSLMTLSTKTPKFFT NHTLVLHVARSEMDKVRVFQATRGKLSSKCSVVLGPKWPSHYLMVPG GKHNMDFYVEALAFPDTDFPGLITLTISLLDTSNLELPEAVVFQDSVVFR VAPWIMTPNTQPPQEVYACSIFENEDFLKSVTTLAMKAKCKLTICPEEE NMDDQWMQDEMEIGYIQAPHKTLPVVFDSPRNRGLKEFPIKRVMGG PDFYVTRGPQTGGISGLDSFGNLEVSPPVTVRGKEYPLGRILFGDSCYP SNDSRQMHQALQDFLSAQQVQAPVKLYSDWLSVGHVDEFLSFVPAPDR KGFRLLLASPRSCYKLFQEQQNEGHGEALLFEGIKKKKQQKIKNILSN KTLREHNSF VERCIDWNRELLKRELGLAESDIIDIPQLFKLKEFSKAEAF FPNMVNMLVLGKHLGIPKPFGPVINGRCCLEEKVCSLLEPLGLQCTFIN DFFTYHIRHGEVHCGTNVRRKPFSFKWWNMVP

b

P-Y motifs of mouse PAD4

MAQGAVIHVAPEQPTHAVCVVGTATPLDVRGSAPKGYTTFGITASPGVI VDVIHGPPVKKSTMGASKWPLDPELEVTLQVKAASSRTDDEKVRVSYY GDKTSPVQALIVITGVELSLSADVTRTGRVKPAQAGKDQSTWTWGPGGRG GPKTSPVQALIYITGVELSLSADVTRTGRVKPAQAGKDQSTWTWGPGGRG AILLVNCDKEDPQASGMDFEDDKILDNKDLQDMSPMTLSTKTPKDFFEKY
QLVLEVPKAKMNRVRVRATRGKLPSRYKVALGPQQFSYCLLPGEQH STDFYVEGLAFPDADFKGLIPLTISLLDKSNPELPEALVFQDSVTFRVAPW IMTPNTQPPQEVY VCRVDNEDFLKSLATLTKKAKCKLTVCEEENIDD QWMQDEMEIGYIQAPHKTLPVVFDPRDRGLKDFPVKRVMGPNFGY TRKLYMSELTGLDAFGNLEVSPPVTVRGKEY LLGRLIGNSGSSSESRD MHQALQDFLSAQQVQAPVRLFSDWLFVGHVDERLSFVPARDKQGFRLL LSSPRACYQLFQELQSQGHGEATLFEGLKRKRQTINEILSNKKLRDQNA YVESCIDWNRAVLKRELGLAEGDIIDIPQLFKLAGNSRGNSKAQAFFPN
MVNMLVLGKYLGIPKPFGPIIDGHCCLEEEVRSHLEPLGLHCTFINDFY MVNMLVLGKYLGIPKPFGPIDGHCCLEEEVRS
TYHVYNGEVHCGTNVRRKPFTFKWWHMVP

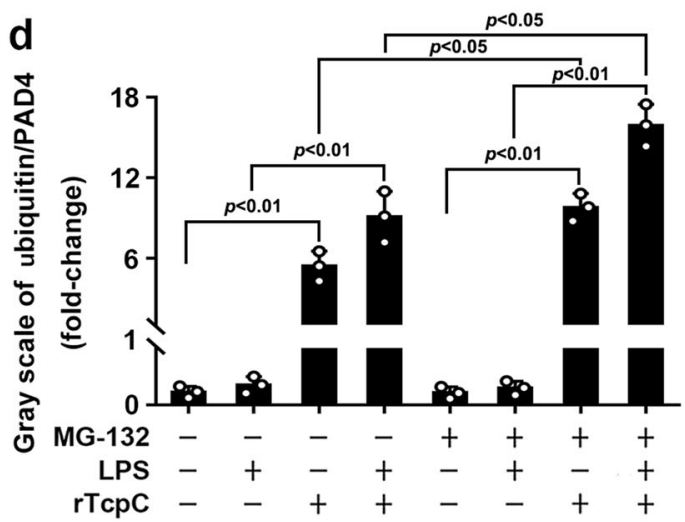

C

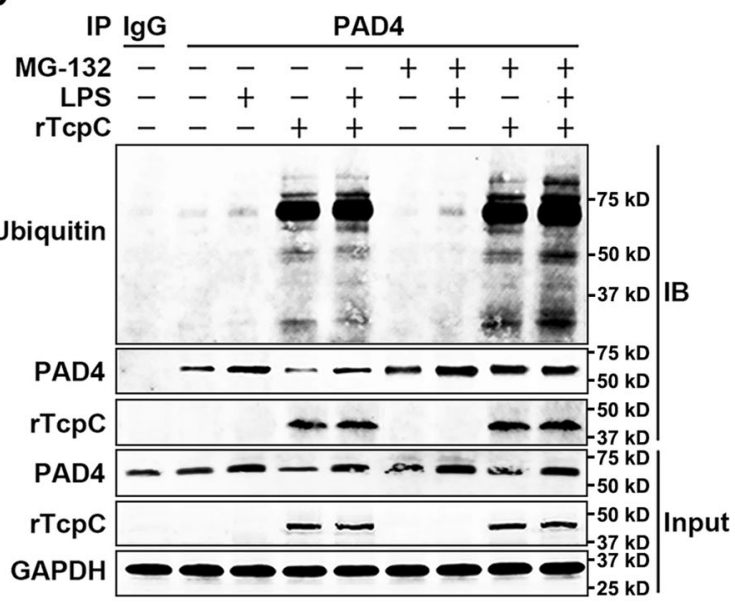

E1 enzym

E2 enzyme

GST-MURF (E3)

S5a (Substrate)

Nutlin-3

rTcpC

Neutrophil lysates

Hu-rPAD4

$\mathrm{Mg}^{2+}$-ATP

Ubiquitin

$++++++++$

$++++++++$

$+++---c_{-}-$

$+++-----$

$-\quad+\quad-\quad+-\frac{1}{+}$

$-\quad-\quad+++++$

$-\quad-+++--$

$-\quad-\quad-\quad-+++$

$-++-++-+$

Fig. 6 TcpC is an E3 ligase that promotes ubiquitination of PAD4. a, b Bioinformatics prediction of ubiquitination functional sites in amino acid sequences of human PAD4 (NCBI Reference Sequence: BAA84542.1) and mouse PAD4 (NCBI Reference Sequence: NP_035191.2). c Co-immunoprecipitation and immunoblotting analyses of ubiquitination of PAD4 in human neutrophils. Western blot data are representative of three independent experiments, $n=3$. d Gray scale analyses of the ubiquitin/PAD4 from experiments as described in $\mathbf{c}$. Mean \pm SD of three independent experiments were shown. $p<0.05$ and $p<0.01$ were considered to be statistically significant and extremely significant respectively. e In vitro ubiquitination kit to examine the PAD4-targeted E3 activity of $r \mathrm{TcpC}$, western blot data is representative of three independent experiments, $n=3$. $p$-values were derived by Dunnett and Mann-Whitney multiple comparison tests. All western blots in panels $\mathbf{c}, \mathbf{e}$ are provided as uncropped blots in Gels and Blots of Source Data file. Source data for panel $\mathbf{d}$ are provided in the separate Source Data file.

influence of TcpC on expression of ROS and proinflammatory cytokines as well as PAD4. We found that rTcpC profoundly inhibited LPS-induced production of ROS (Fig. 2d-f) and expression of proinflammatory cytokines at both protein and mRNA levels (Fig. 2g, h) in neutrophils. But unlike the influence on proinflammatory cytokines, rTcpC suppressed, in a dose- and time-dependent manner, protein (Fig. 5b, c, e, f) but not mRNA (Fig. 5a, d) levels of PAD4, suggesting that $\mathrm{rTcpC}$ decreases PAD4 protein by post-transcriptional modification. Because level of $\mathrm{CitH} 3$ is determined by PAD4 and rTcpC decreases concentration of PAD4 in neutrophils, it is no wonder that $\mathrm{rTcpC}$ itself can dynamically suppress levels of $\mathrm{CitH3}$ (Fig. 3d).

It has been demonstrated that ubiquitination plays an important role in protein post-translational modification in eukaryotic cells that regulates many biological processes ${ }^{39,48,55-57}$, and in many aspects of the immune system, including innate and adaptive immunity and antimicrobial autophagy. In addition, increasing evidence indicates that microbial pathogens exploit the ubiquitin pathway to evade the host immune system ${ }^{58,59}$. Furthermore, our previous studies have demonstrated that TcpC is an E3 ubiquitin ligase of HECT family ${ }^{45}$, the influence of rTcpC on ubiquitination of PAD4 was examined. Our data clearly show that rTcpC treatment caused significantly enhanced ubiquitination of PAD4 in neutrophils (Fig. 6c, d). Bioinformatics analyses showed that in the amino acid sequence of human (Fig. 6a) and mouse (Fig. 6b) PAD4 there are P-Y motifs of the E3 ligase substrates, suggesting that TcpC might be a PAD4-targetd E3 ubiquitin ligase. This speculation was further verified by in vitro ubiquitination kit assays. rTcpC could serve as the E3 to promote ubiquitination of both proteins in the lysate of neutrophils and Hu-rPAD4, and this E3 activity of rTcpC could be abrogated by the E3 inhibitor Nutlin-3 (Fig. 6e). These data confirm that TcpC is a PAD4-targeted E3 ubiquitin ligase.

To examine whether $\mathrm{rTcpC}$ treatment promotes accumulation of PAD4 in proteasome. Co-localization of PAD4 with the proteasome maker PSMD2 in neutrophils was observed by confocal microscopy. rTcpC treatment led to increased accumulation of PAD4 in proteasomes in LPS-treated neutrophils (Fig. 7a-c). Particularly, when the proteasome activity was blocked by MG132, accumulation of PAD4 in proteasome increased steadily 

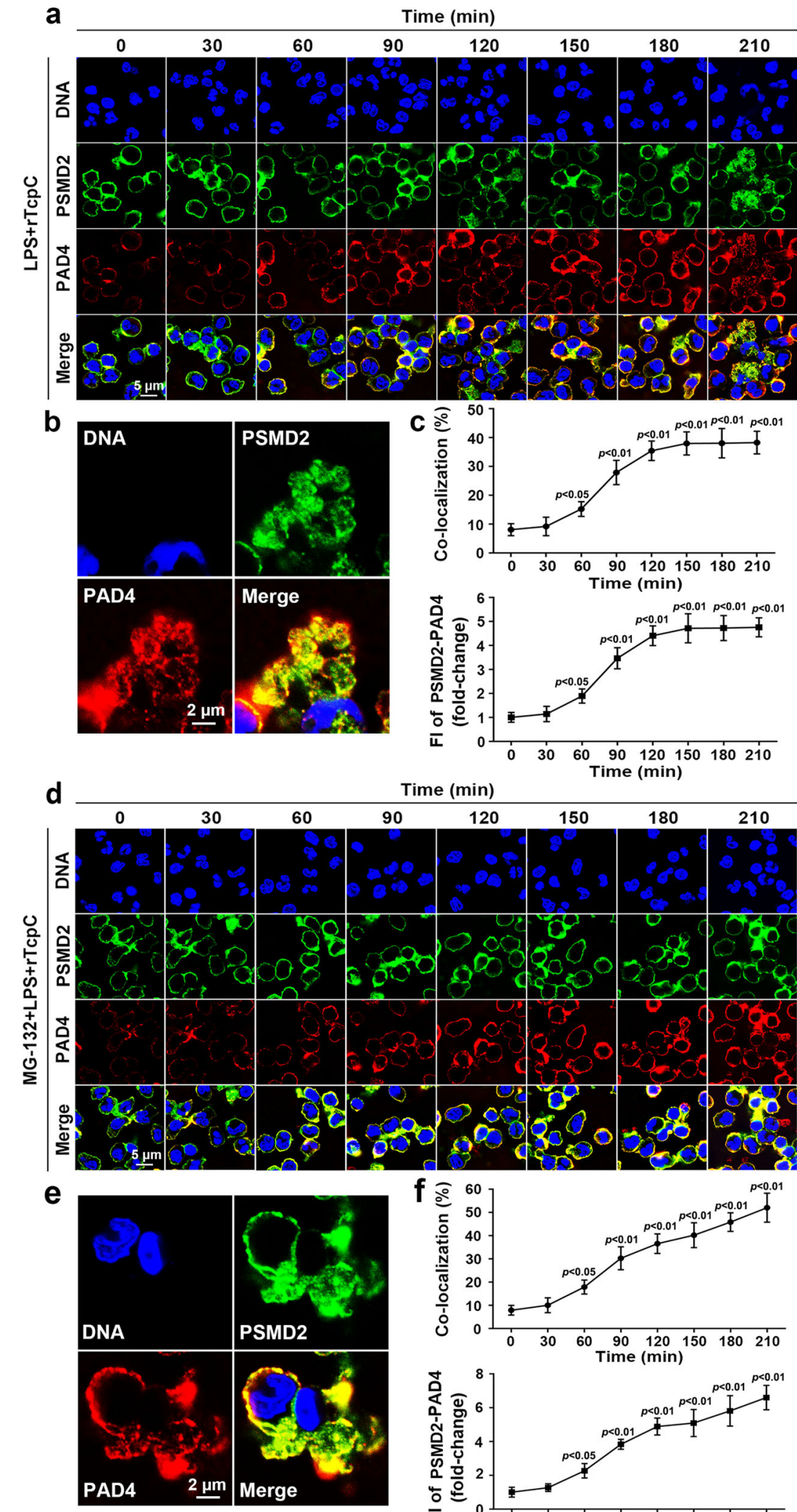

Time $(\min )$

during the whole treatment period (Fig. $7 \mathrm{~d}-\mathrm{f}$ ). These data confirm that TcpC promotes degradation of PAD4 through ubiquitin-proteasome pathway.

At last, we examined the influence of rTcpC-C12S on degradation of PAD4 and LPS-induced NETosis. In line with the changes of activity in degrading PAD4 (Fig. 8a, b), rTcpC-C12S

showed significantly decreased activity to inhibit the LPS-induced NETosis compared with the rTcpC prototype (Fig. 8c-e). These data suggest that the inhibitory effect of TcpC on NETosis is primarily mediated by promoting ubiquitinated degradation of PAD4.

In summary, this study demonstrated that $\mathrm{TcpC}$ inhibits NETosis by serving as a PAD4-targeted E3 ubiquitin ligase that 
Fig. 7 rTcpC treatment leads to accumulation of PAD4 in proteasomes. a Dynamic observation of co-localization of PAD4 with PSMD2 by confocal microscopy. Scale bar $=5 \mu \mathrm{m}$. b Enlarged image of the co-localization in $\mathbf{a}$ at $210 \mathrm{~min}$. Scale bar $=2 \mu \mathrm{m}$. Confocal microscopy images are representative of three independent experiments from three donors' neutrophils, $n=3$. c Percent and Fl of co-localization were analyzed by ImageJ software. Mean \pm SD of three independent experiments as described in a were shown. $p<0.05$ and $p<0.01$ compared with the group at 0 min. $\mathbf{d}$ Dynamic observation of colocalization of PAD4 with PSMD2 in the presence of MG-132 by confocal microscopy. Scale bar $=5 \mu \mathrm{m}$. e Enlarged image of the co-localization in $\mathbf{d}$ at 210 min. Scale bar $=2 \mu \mathrm{m}$. Confocal microscopy images are representative of three independent experiments from three donors' neutrophils, $n=3$. $\mathbf{f}$ Percent and $\mathrm{Fl}$ of co-localization were analyzed by ImageJ software. Mean \pm SD of three independent experiments as described in $\mathbf{d}$ were shown. $p<0.05$ and $p<$ 0.01 compared with the group at 0 min. $p$-values were derived by Dunnett test. Source data for panel $\mathbf{c}$ and $\mathbf{f}$ are provided in the separate Source Data file.
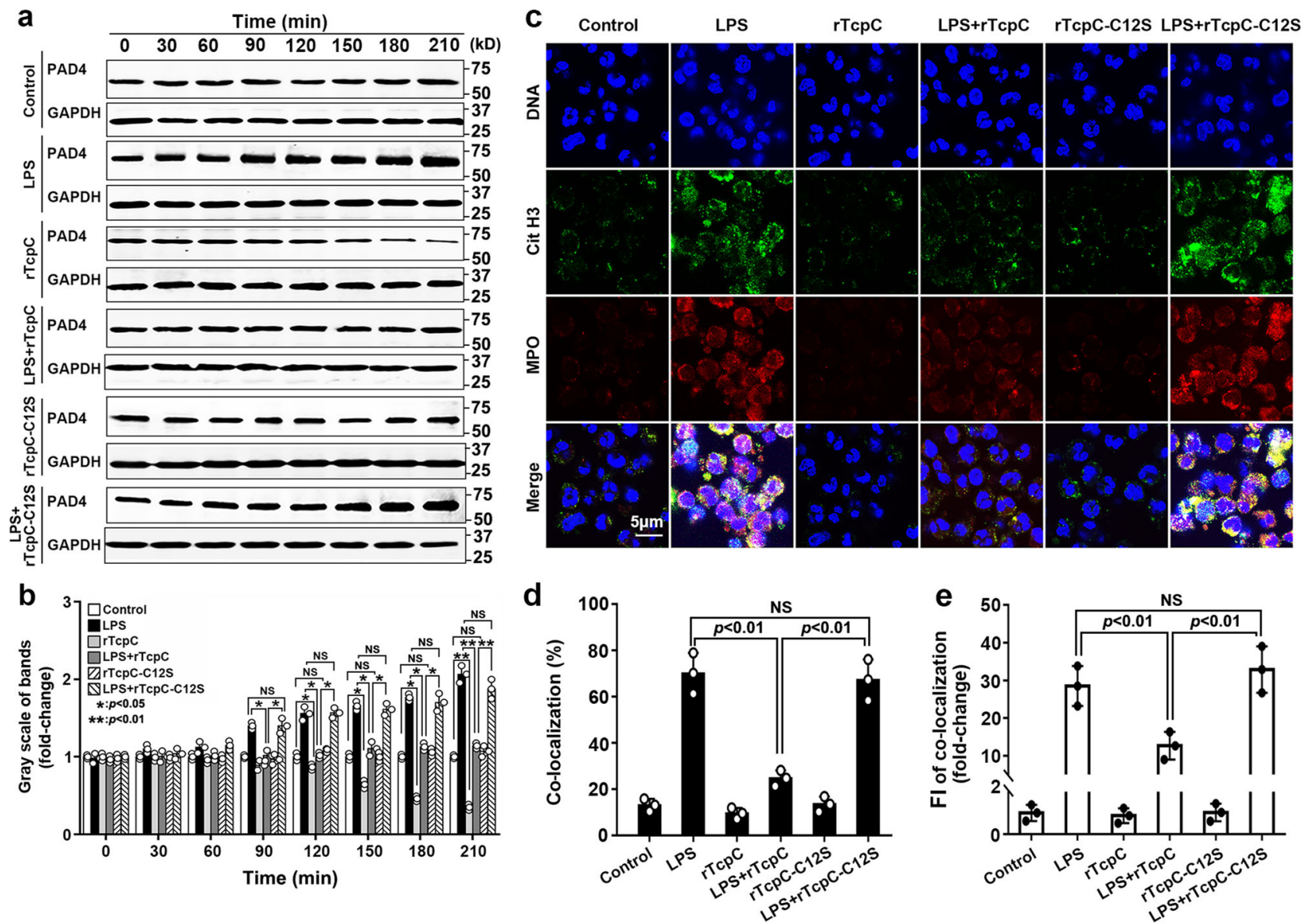

Fig. 8 rTcpC-C12S loses ability to promote degradation of PAD4 and inhibit LPS-induced NETosis. a Western blot analyses to detect the ability of rTcpC-C12S to promote rPAD4 degradation. One representative of three independent experiments was shown, $n=3$. $\mathbf{b}$ Gray scale analyses of bands from experiments as described in a. Gray scale values reflecting the PAD4 levels in the control group were set as 1.0. Mean \pm SD of three independent experiments were shown. $p<0.05$ and $p<0.01$ were considered to be statistically significant and extremely significant respectively, NS not significant. c Confocal microscopy to detect the ability of $\mathrm{rTcpC}-\mathrm{C} 12 \mathrm{~S}$ to inhibit LPS-induced NETosis. Scale bar $=5 \mu \mathrm{m}$. $\mathbf{d}$ Percent of co-localization was analyzed by Image J software. Mean \pm SD of three independent experiments as described in $\mathbf{c}, p<0.01$ was considered to extremely significant. e FI of co-localization was analyzed by ImageJ software. Fl in control group was set as 1.0. Mean \pm SD of three independent experiments as described in $\mathbf{c}$ were shown, $n=3, p<$ 0.01 was extremely significant. All western blots in panels a are provided as uncropped blots in Gels and Blots of Source Data file. $p$-values were derived by Dunnett and Mann-Whitney multiple comparison tests. Source data for panel $\mathbf{b}, \mathbf{d}$ and $\mathbf{e}$ are provided in the separate Source Data file.

promote degradation of PAD4 via ubiquitin-proteasome pathway (Fig. 9). Our findings provide not only a novel mechanism by which TcpC-secreting UPECs evade host innate immune response, but also new clues to clarify the pathogenicity of other microbial pathogens.

\section{Methods}

Ethics statement. Animal experiments were performed in accordance with the National Regulations for the Administration of Experimental Animals of China (1988-002) and the National Guidelines for Experimental Animal Welfare of China (2006-398). All the animal experimental protocols were approved by the Ethics Committee for Animal Experiment of Zhejiang University City College
School of Medicine and fully informed consent of all healthy blood donors was obtained after clearing the possible consequences of the study.

Reagents. Fetal bovine serum (FBS, Gibco, USA, 10100147), RPMI 1640 medium (Gibco, USA, 22400105), LPS (Sigma, USA, L2630), Sytox Green (Invitrogen, USA S7020), IL-1 $\beta$, IL-6, TNF- $\alpha$ ELISA Kit (eBioscience, USA, KAC1211, EH2IL6, KHC3011), DCFH-DA probe dye (Thermo, USA, I36007), BCA Protein Concentration Kit (Beyotime, China, P0012S), rabbit anti-PAD4-IgG (Abcam, UK, ab96758), rabbit anti-MPO-IgG (Abcam, UK, ab208670), rabbit anti-ubiquitin-IgG (Abcam, UK, ab134953), anti-GAPDH-IgG (Abcam, UK, ab181602), AntiPSMD2-IgG (Abcam, UK, ab125914), Alexa fluor $647 \mathrm{~nm}$ goat anti-rabbit IgG (Invitrogen, USA, A-21244) and Alexa fluor $488 \mathrm{~nm}$ goat anti-mouse IgG fluorescent secondary antibody (Invitrogen, USA, A-11001), DAPI (Invitrogen, USA, 


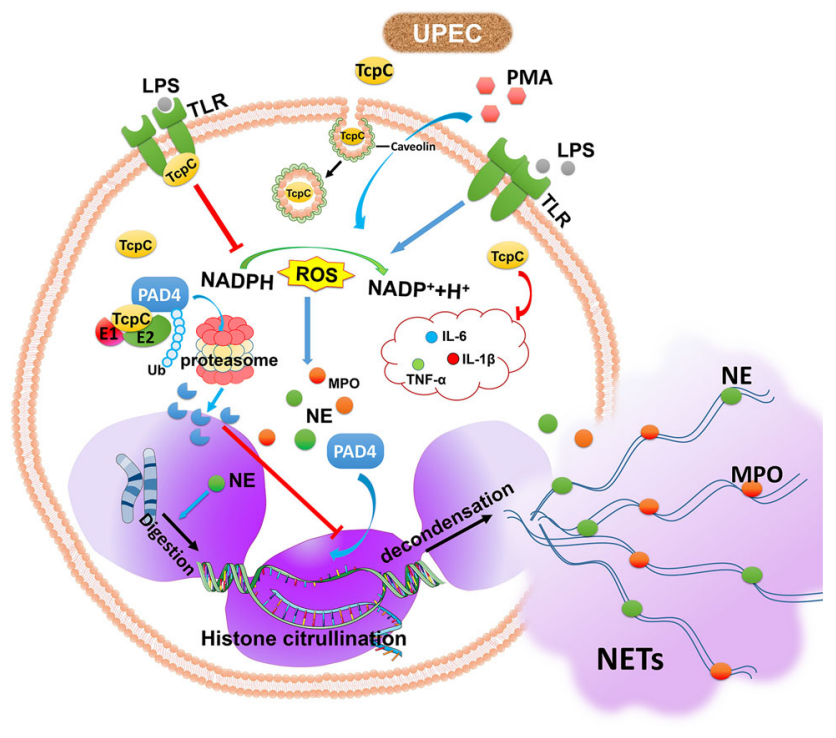

Fig. 9 Schematic diagram of the mechanisms underlying TcpC-mediated inhibition of NETosis. Upon stimulation of LPS and PMA, NADPH oxidase catalyzes the intracellular $\mathrm{O}_{2}$ to generate ROS. ROS activate downstream NETosis essential enzyme, such as MPO, NE, and PAD4. NEs digest nucleosomal histones and promote chromatin relaxation, which favors PAD4 to catalyze histone into citrullinated histone. Citrullination of histones causes chromatin entropic swelling and release of NETs. In case of TcpC-secreting UPEC infection, TcpC inhibits pathogen-induced NETosis via two mechanisms: (1) Inhibition of ROS production, and (2) Functioning as an E3 ubiquitin ligase that promotes PAD4 degradation through ubiquitin-proteasome pathway.

D1306), Ly-6G/Ly-6C (PE, Thermo, USA, 14-5931-81), CD11b (FITC, Thermo, USA, 11-0112-41), Nutlin-3 (Selleckchem, USA, S1061), MG-132 (Selleckchem, USA, S2619), primer (Invitrogen, USA), RNAiso Plus (TaKaRa, Japan, 9109), PrimeScript ${ }^{\mathrm{TM}}$ RT reagent Kit with gDNA Eraser (TaKaRa, Japan, RR047A), TB Green TM Premix Ex TaqTM (TaKaRa, Japan, RR071A), Pierce ${ }^{\mathrm{TM}}$ Protein A/G magnetic beads (Thermo, USA, 88802), MuRF1 Ubiquitin Ligase Kit-S5a Substrate (Boston Biochem, USA, K-102), rabbit anti-Ly-6G-IgG (Abcam, UK, ab238132), Alexa fluor $546 \mathrm{~nm}$ goat anti-rabbit IgG (Thermo, USA, A-11035), rabbit antiCitH3-IgG (Abcam, UK, ab5103), Alexa fluor $488 \mathrm{~nm}$ goat anti-rabbit IgG fluorescent secondary antibody (Invitrogen, USA, A-11008), ProLong Diamond Antifade Mountant (Thermo, USA, P36990), Dynasore (MCE, USA, HY-15304), TAK242 (MCE, USA, HY-11109), Methyl- $\beta$-cyclodextrin (MCE, USA, HY-101461), and recombinant TcpC protein and rabbit anti-TcpC-IgG (made in our laboratory).

Mouse PN models and in situ NETosis examination. Female C57BL/6 mice, 6-8 weeks of age, were provided by Shanghai Slack Laboratory Animals Co., Ltd (Shanghai, China). PN murine models were induced by transurethral instillation of CFT073 ${ }^{\text {wt }}$ or CFT073 ${ }^{\Delta t c p c}$ as described in our previous report ${ }^{22}$. Normal salinetreated mice served as the control group. In all, $72 \mathrm{~h}$ later, the model mice were sacrificed and kidneys were removed for pathological and in situ NETosis examinations as described previously ${ }^{60}$. After the sections were fixed and tissue antigen repairing, the fixed tissues were incubated with rabbit anti-Ly-6G-IgG, rabbit antiCitH3-IgG, and rabbit anti-MPO-IgG (Abcam, UK, 1:200 dilution) overnight at $4{ }^{\circ} \mathrm{C}$ and stained with different fluorescent secondary antibody (Alexa fluor $546 \mathrm{~nm}$ goat anti-rabbit IgG, Alexa fluor $488 \mathrm{~nm}$ goat anti-rabbit IgG and Alexa fluor 647 $\mathrm{nm}$ goat anti-rabbit IgG, 1:500 dilution) in dark respectively, then added ProLong Diamond Antifade Mountant (Thermo, USA) and DAPI to each coverslip, the coverslips were placed on the slides and confocal microscope images were taken.

Histological examination. Kidneys were fixed in freshly prepared $4 \%$ paraformaldehyde soon after dissection and incubated overnight at $4{ }^{\circ} \mathrm{C}$. The fixed tissues were incubated in $15 \%$ sucrose at $4^{\circ} \mathrm{C}$ and washed in $25 \%$ ice cold sucrose at $4{ }^{\circ} \mathrm{C}$. Tissues were then frozen in isopentane at $-80^{\circ} \mathrm{C}$. Cryostat sections were made, mounted onto poly-D-lysine-coated glass slides and stained with htx-eosin ${ }^{21}$.

Isolation of human neutrophils. EDTA-anticoagulated peripheral blood from healthy donors was used to isolate neutrophils using EasySep Direct Human Neutrophil Isolation Kit (StemCell Technologies, USA) following the manufacturer's instructions. Erythrocytes were lysed with red blood cell lysis buffer and washed with PBS. Subsequently, purity of the human neutrophils was evaluated by FACS using antibodies against Ly-6G/Ly-6C (1:500 dilution, Ly-6G/Ly-6C monoclonal antibody, PE, Thermo, USA) and CD11b (1:500 dilution, CD11b monoclonal antibody, FITC, Thermo, USA). Neutrophil purity was $>93 \%$ (Supplementary Fig. 4).

Transwell co-culture. Neutrophils $\left(1 \times 10^{6}\right)$ were separately co-cultured for $12 \mathrm{~h}$, in transwell (Corning, USA) at a multiplicity of infection (MOI) $=100$, with CFT073 ${ }^{\mathrm{wt}}$ or CFT073 ${ }^{\Delta t c p c}$. Neutrophil cultures without E. coli treatment served as the control group. In all, $4 \mathrm{~h}$ before the end of the culture, $1 \mu \mathrm{g} / \mathrm{ml}$ LPS was added into corresponding wells. Then the cells were harvested and subjected to confocal microscopy to examine NETosis.

rTcpC treatment of neutrophils. In all, $1 \times 10^{6}$ neutrophils were treated, in the presence or absence of $1 \mu \mathrm{g} / \mathrm{ml}$ LPS, $100 \mathrm{nM}$ PMA, with or without $20 \mu \mathrm{M} \mathrm{TAK}$ $242,4 \mu \mathrm{g} / \mathrm{ml} \mathrm{rTcpC}$ for $210 \mathrm{~min}$. Neutrophils cultured in medium alone was set as control group. Culture supernatants were collected for measurement of proinflammatory cytokines and cells were subjected to detection of NETosis, qRT-PCR analyses of cytokine and pad4 mRNA and western blot analyses of PAD4, respectively.

Confocal microscopy to examine NETosis. The above treated neutrophils were fixed by $4 \%$ paraformaldehyde in PBS overnight at $4{ }^{\circ} \mathrm{C}$, then permeabilized with $0.1 \%$ TritonX-100 in PBS for $20 \mathrm{~min}$, and washed three times with PBS. Cells were blocked with $1 \%$ BSA, $0.1 \%$ Tween 20 in PBS for $2 \mathrm{~h}$ at room temperature, and then incubated with rabbit anti-MPO antibodies (Abcam, UK, 1:200 diluted in $0.1 \%$ Tween 20 in PBS) overnight at $4^{\circ} \mathrm{C}$. Next, neutrophils were gently washed three times with PBS and incubated in the dark with goat anti-rabbit IgG antibody (Alex Flour $647 \mathrm{~nm}, 1: 500$ diluted in the PBS) for $2 \mathrm{~h}$ at room temperature. Before staining with $100 \mathrm{ng} / \mathrm{ml}$ DAPI, neutrophils were incubated with rabbit anti-CitH3 antibodies (Abcam, UK, 1:200 diluted in $0.1 \%$ Tween 20 in PBS) overnight at $4{ }^{\circ} \mathrm{C}$, then washed three times and incubated with Alexa fluor $488 \mathrm{~nm}$ goat anti-rabbit IgG fluorescent secondary antibody, finally washed with PBS and ProLong Diamond Antifade Mountant (Thermo, USA) was added to each coverslip, the coverslips were placed on the slides, and then observed with a confocal fluoresce microscope (FV3000 Olympus, Japan). Images were analyzed with the ImageJ software from National Institutes of Health ${ }^{33,61}$.

Quantification of NETs. NETs formation was quantified by detecting DNA release spectrophotometrically with the DNA-binding dye Sytox Green as previously described ${ }^{33}$. Briefly, neutrophils were treated for $210 \mathrm{~min}$ with or without $4 \mu \mathrm{g} / \mathrm{ml}$ $\mathrm{rTcpC}$ in the presence or absence of $1 \mu \mathrm{g} / \mathrm{ml}$ LPS, at the beginning of the incubation $500 \mathrm{nM}$ Sytox Green were added into each well. Fluorescence values were measured every $30 \mathrm{~min}$ with the $488 \mathrm{~nm}$ excitation. Scale was set and threshold adjusted to define the area of fluorescent DNA. Total area of all fluorescent particles indicated the amount of NETs formation. Unstimulated neutrophils had an area of $62 \pm 6.1 \mu \mathrm{m}^{2}$. Thus only particles $>70 \mu \mathrm{m}^{2}$ were considered NETs.

Confocal microscopy to detect ROS production. In all, $1 \times 10^{6} / \mathrm{ml}$ neutrophils were treated with or without $4 \mu \mathrm{g} / \mathrm{ml} \mathrm{TcpC}$ and $1 \mu \mathrm{g} / \mathrm{ml}$ LPS for $210 \mathrm{~min}$. Cells were harvested and resuspended in $1 \mathrm{ml}$ serum-free culture medium. Cells were stained with $1 \mu \mathrm{l}$ DCFH-DA probe dye (Invitrogen, USA) and $100 \mathrm{ng}$ DAPI (Invitrogen, USA) in dark for $20 \mathrm{~min}$ at $37^{\circ} \mathrm{C}$. After three times wash with PBS, cells in each group were adjusted to a density of $1 \times 10^{6}$ cells $/ \mathrm{ml}$. In total, $10 \mu \mathrm{l}$ cells were put on a Poly-D-Lys (Sigma, USA) pre-coated coverslip. In all, 1 min later the coverslip was put on a slide and observed with a confocal microscope (FV3000 Olympus, Japan). Images were analyzed with the ImageJ software.

ELISA. Concentrations of IL-1 $1 \beta$, IL- 6 , and TNF- $\alpha$ in supernatants of neutrophils were measured by ELISA according to the instructions of the manufacturer (eBioscience, USA).

qRT-PCR. Total RNA of treated neutrophils was extracted by RNAiso Plus (TaKaRa, Japan), and cDNA was synthesized by reverse transcription using PrimeScript ${ }^{\mathrm{TM}}$ RT reagent kit with gDNA Eraser (TaKaRa, Japan). The mRNA levels of IL-1 $\beta$, IL-6, TNF- $\alpha$, and PAD4 in neutrophils were detected by qRT-PCR, and $\beta$ actin was set as an internal reference. The primers are listed in Table 1 . The Cq value was calculated by $2^{-\Delta \Delta C T}$ method.

Flow cytometry analyses of ROS. The ROS generation during NETosis was detected by FACS using DCFH-DA (Invitrogen, USA). Before stimulation, neutrophils were preloaded with DCFH-DA for 20 min, cells were washed three times with PBS. Then, neutrophils were treated with LPS or rTcpC for 210 min. Finally, the fluorescence signals of DCFH-DA were read at FITC channel on a BD FACSCalibur. 
Table 1 Information of primers used in this study.

\begin{tabular}{lll} 
Primer & Sequence $\left(\mathbf{5}^{\prime}\right.$ to $\mathbf{3}^{\prime}$ ) & Purpose \\
\hline pad4 & F: CTCTCCAGGAGTCATCGTAG & Detection of \\
& R: CCAACACCAGCTGATACTTT & pad4 mRNA \\
$i l-1 \beta$ & F: CCTTGTGCAAGTGTCTGAAG & Detection of $i$ - \\
& R: GGGCTTGGAAGCAATCCTTA & $1 \beta$ mRNA \\
il-6 & F: GCCCTTCAGGAACAGCTATGA & Detection of $i$ - \\
& R: TGTCAACAACATCAGTCCCAAGA & 6 mRNA \\
tnf- $\alpha$ & F: CCTGTAGCCCACGTCGTAG & Detection of tnf- \\
& R: GGGAGTAGACAAGGTACAACCC & $\alpha$ mRNA \\
$\beta$-actin & F: ATGGATGACGATATCGCTG & Inner reference \\
& R: AACACCCATTCCCTTCACAG & \\
\hline \multirow{2}{*}{ forward primer, $R$ reverse primer. } & \\
\hline
\end{tabular}

Western blot analyses of PAD4. Proteins in supernatants of lysates from treated neutrophils were prepared. After $12 \%$ SDS-PAGE, proteins were electrotransferred onto PVDF membranes. Proteins were probed with rabbit anti-PAD4 $\operatorname{IgG}\left(1: 10000\right.$ dilution, Abcam, UK) as primary antibody and IRDye ${ }^{\circledast}$ 680RD goat anti-rabbit-IgG $(\mathrm{H}+\mathrm{L})(1: 5000$ dilution, LI-COR, USA $)$ as secondary antibody. Images were developed using ODYSSEY ${ }^{\circledR}$ CLx Infrared Imaging System (LI-COR). Gray scale values of bands were analyzed by ImageJ software.

Dynamic analyses of CitH3 levels. Neutrophils $\left(1 \times 10^{6}\right)$ were treated with or without $4 \mu \mathrm{g} / \mathrm{ml} \mathrm{rTcpC}$ for the indicated time in the absence or presence of $1 \mu \mathrm{g} / \mathrm{ml}$ LPS. Proteins in supernatants of lysates from treated neutrophils were prepared. After 12\% SDS-PAGE, proteins were electro-transferred onto PVDF membranes. Proteins were probed with rabbit anti-CitH3 IgG (1:5000 dilution, Abcam, UK) as primary antibody and IRDye $^{\circledast}$ 680RD goat anti-rabbit-IgG $(\mathrm{H}+\mathrm{L})(1: 5000$ dilution, LI-COR, USA) as secondary antibody. Images were developed using ODYSSEY ${ }^{\circledR}$ CLx Infrared Imaging System (LI-COR). Gray scale values of bands were analyzed by ImageJ software.

\section{Co-immunoprecipitation and immunoblotting to detect ubiquitination of}

PAD4. The treated neutrophils were washed twice with PBS and were lysed with RIPA. After centrifugation at $12,000 \times \mathrm{g}$ for $30 \mathrm{~min}$ at $4{ }^{\circ} \mathrm{C}$, the supernatants were collected and protein concentrations were determined by a BCA Protein Assay Kit (Beyotime, China). Proteins in cell lysates were then immunoprecipitated with the rabbit anti-PAD4 IgG (1:1000 dilution, Abcam, UK) using Pierce ${ }^{\mathrm{pm}}$ Protein A/G Magnetic Beads (Thermo Scientific) and the released proteins (rTcpC, PAD4 and ubiquitin) were detected by immunoblotting. In detection of ubiquitin and rTcpC, rabbit anti-ubiquitin IgG (1:1000 dilution, Abcam, UK) and the self-made rabbit anti-rTcpC IgG were used as the primary antibody respectively, and IRDye ${ }^{\circledast}$ 680RD goat anti-rabbit-IgG $(\mathrm{H}+\mathrm{L})$ (1:5000 dilution, LI-COR, USA) as secondary antibody.

In vitro ubiquitination kit assays. MuRF1 Ubiquitin Ligase Kit-S5a Substrate (Boston Biochem, USA) was used to detect ubiquitination of PAD4 and determine the E3 ligase function of rTcpC. When the E3 was replaced by $\mathrm{rTcpC}$, proteins in the neutrophil lysates and rPAD4 (Abcam, UK) were used as the substrates. All the experiments were performed according to instructions of the manufacturer. Ubiquitination of PAD4 was detected by western blotting as described above.

Confocal microscopy to examine co-localization of PAD4 with PSMD2. Neutrophils were treated with or without $4 \mu \mathrm{g} / \mathrm{ml} \mathrm{rTcpC}$ and $1 \mu \mathrm{g} / \mathrm{ml}$ LPS for the indicated time, cells were collected and fixed with $4 \%$ paraformaldehyde overnight at $4{ }^{\circ} \mathrm{C}$. The fixed cells were permeabilized with $0.1 \%$ TritonX-100 in PBS for 20 $\mathrm{min}$, and washed three times with PBS. After blocked with $1 \%$ BSA, $0.1 \%$ Tween 20 in PBS for $2 \mathrm{~h}$ at room temperature, cells were stained with rabbit anti-PAD4 IgG ( $1: 100$ dilution, Abcam, UK) overnight at $4{ }^{\circ} \mathrm{C}$, and then probed with the secondary antibody Alex Flour 647 goat anti-rabbit IgG fluorescence antibody (1:500 dilution, Invitrogen, USA) for $2 \mathrm{~h}$ at room temperature in dark. After three times wash with PBS, the cells were also probed with rabbit anti-PSMD2 (1:100 dilution, Abcam, UK) and Alexa Fluor 488 goat anti-rabbit IgG fluorescent antibody (1:200 dilution, Invitrogen, USA) as primary and secondary antibodies respectively. Neutrophils were finally washed with PBS and stained with $100 \mathrm{ng} / \mathrm{ml}$ DAPI for $30 \mathrm{~min}$. Colocalization of PAD4 with PSMD2 was observed by confocal microscopy (Olympus) $(495 / 519,652 / 668$ or $485 \mathrm{~nm}$ excitation/emission wavelengths for Alexa Fluor 488, Alexa Fluor 647 or DAPI detection). Percentages and yellow FI reflecting the co-localization were analyzed by ImageJ software ${ }^{62}$. To examine the influence of MG-132 on rTcpC induced accumulation of PAD4 in proteasomes, neutrophils were treated with $1 \mu \mathrm{M}$ MG-132 for 30 min before treatment with $4 \mu \mathrm{g} / \mathrm{ml} \mathrm{rTcpC}$ for the indicated time, and then the treated cells were subjected to confocal microscopy as described above.
Transcriptome sequencing. Neutrophils $\left(1 \times 10^{6}\right.$ cells $)$ were treated for $120 \mathrm{~min}$ with $1 \mu \mathrm{g} / \mathrm{ml} \mathrm{LPS}, 4 \mu \mathrm{g} / \mathrm{ml} \mathrm{rTcpC}, 4 \mu \mathrm{g} / \mathrm{ml} \mathrm{rTcpC}+1 \mu \mathrm{g} / \mathrm{ml}$ LPS, respectively, and untreated neutrophils were served as the control group. Cells were collected (1500 $\mathrm{rpm}, 10 \mathrm{~min}$ ) and frozen at $-80^{\circ} \mathrm{C}$ with RNAiso Plus (TaKaRa, Japan). Total RNA was extracted and a genome-wide transcriptomics analysis was conducted (LCBIOTECHNOLOGIS (HANGZHOU) CO., LTD). The differentially expressed mRNAs were selected with fold change $>2$ or fold change $<0.5$ and $p$-value $<0.05$ by $R$ package edgeR (https://bioconductor.org/packages/release/bioc/html/edgeR.html) or DESeq2 (http://www.bioconductor.org/packages/release/bioc/html/DESeq2.html).

Bioinformatics analyses. The structure and functional domains in PAD4 of human and mouse were analyzed using NCBI-Batch CD-Search software ${ }^{63}$.

Statistics and reproducibility. Data shown are mean \pm SD of three independent experiments. Images were analyzed by ImageJ software. Dunnett and Mann-Whitney tests of SPSS 22.0 were used for variance analysis. Data from quantification of NETs formation were analyzed by Mann-Whitney test. $p<0.05$ is considered to be statistically significant and $p<0.01$ is extremely significant. NS is considered to be not significant. All source data are provided in separate Raw Data file of Source Data file.

Reporting summary. Further information on experimental design is available in the Nature Research Reporting Summary linked to this paper.

\section{Data availability}

Data supporting the findings of this manuscript are available from the corresponding author upon request. Full scan images of the Gels and Blots and Source data for figures and numbers are provided with this paper. We deposited our data including the transcriptomic data in GEO DataSets, the accession number is GSE173807. Source data are provided with this paper.

Received: 1 August 2020; Accepted: 24 May 2021; Published online: 09 June 2021

\section{References}

1. Sihra, N., Goodman, A., Zakri, R., Sahai, A. \& Malde, S. Nonantibiotic prevention and management of recurrent urinary tract infection. Nat. Rev. Urol. 15, 750-776 (2018).

2. McLellan, L. K. \& Hunstad, D. A. Urinary tract infection: pathogenesis and outlook. Trends Mol. Med. 22, 946-957 (2016).

3. Gaitonde, S., Malik, R. D. \& Zimmern, P. E. Financial burden of recurrent urinary tract infections in women: a time-driven activity-based cost analysis. Urology 128, 47-54 (2019).

4. Luthje, P. \& Brauner, A. Virulence factors of uropathogenic E. coli and their interaction with the host. Adv. Microb. Physiol. 65, 337-372 (2014).

5. Cirl, C. et al. Subversion of Toll-like receptor signaling by a unique family of bacterial Toll/interleukin-1 receptor domain-containing proteins. Nat. Med. 14, 399-406 (2008)

6. Snyder, G. A. et al. Molecular mechanisms for the subversion of MyD88 signaling by TcpC from virulent uropathogenic Escherichia coli. Proc Natl Acad. Sci. USA 110, 6985-6990 (2013).

7. Waldhuber, A. et al. Uropathogenic Escherichia coli strain CFT073 disrupts NLRP3 inflammasome activation. J. Clin. Invest. 126, 2425-2436 (2016).

8. de Bont, C. M., Koopman, W. J. H., Boelens, W. C. \& Pruijn, G. J. M Stimulus-dependent chromatin dynamics, citrullination, calcium signalling and ROS production during NET formation. Biochim. Biophys. Acta Mol. Cell Res. 1865, 1621-1629 (2018).

9. Neubert, E. et al. Chromatin swelling drives neutrophil extracellular trap release. Nat. Commun. 9, 3767 (2018).

10. Ruiz-Limon, P. et al. Tocilizumab improves the proatherothrombotic profile of rheumatoid arthritis patients modulating endothelial dysfunction, NETosis, and inflammation. Transl. Res. 183, 87-103 (2017).

11. Kuhnle, A. et al. Polysialic acid interacts with lactoferrin and supports its activity to inhibit the release of neutrophil extracellular traps. Carbohydr. Polym. 208, 32-41 (2019).

12. Perdomo, J. et al. Neutrophil activation and NETosis are the major drivers of thrombosis in heparin-induced thrombocytopenia. Nat. Commun. 10, 1322 (2019).

13. Lewis, H. D. et al. Inhibition of PAD4 activity is sufficient to disrupt mouse and human NET formation. Nat. Chem. Biol. 11, 189-191 (2015).

14. Saunders, C. A. \& Parent, C. A. Emerging roles for the nucleus during neutrophil signal relay and NETosis. Curr. Opin. Cell Biol. 62, 135-143 (2019).

15. Chen, K. W., Demarco, B. \& Broz, P. Beyond inflammasomes: emerging function of gasdermins during apoptosis and NETosis. EMBO J. 39, e103397 (2020). 
16. Franck, G. et al. Roles of PAD4 and NETosis in experimental atherosclerosis and arterial injury. Circ. Res. 123, 33-42 (2018).

17. Guiducci, E. et al. Candida albicans-induced NETosis is independent of peptidylarginine deiminase 4. Front. Immunol. https://doi.org/10.3389/ fimmu.2018.01573 (2018).

18. Li, P. et al. PAD4 is essential for antibacterial innate immunity mediated by neutrophil extracellular traps. J. Exp. Med. 207, 1853-1862 (2010).

19. Douda, D. N., Khan, M. A., Grasemann, H. \& Palaniyar, N. SK3 channel and mitochondrial ROS mediate NADPH oxidase-independent NETosis induced by calcium influx. Proc. Natl Acad. Sci. USA 112, 2817-2822 (2015).

20. Papayannopoulos, V. Neutrophil extracellular traps in immunity and disease. Nat. Rev. Immunol. 18, 134-147 (2018).

21. Yadav, M. et al. Inhibition of TIR domain signaling by TcpC: MyD88dependent and independent effects on Escherichia coli virulence. PLoS Pathog. 6, e1001120 (2010).

22. He, Y. et al. TcpC secreting uropathogenic E. coli promoted kidney cells to secrete MIP-2 via p38 MAPK pathway. Mol. Med. Rep. 16, 3528-3534 (2017).

23. Khan, I. \& Steeg, P. S. Endocytosis: a pivotal pathway for regulating metastasis. Br. J. Cancer 124, 66-75 (2021).

24. McMahon, H. T. \& Boucrot, E. Molecular mechanism and physiological functions of clathrin-mediated endocytosis. Nat. Rev. Mol. Cell Biol. 12, 517-533 (2011).

25. Almeida-Souza, L. et al. A flat BAR protein promotes actin polymerization at the base of clathrin-coated pits. Cell 174, 325-337.e314 (2018).

26. Cullen, P. J. \& Steinberg, F. To degrade or not to degrade: mechanisms and significance of endocytic recycling. Nat. Rev. Mol. Cell Biol. 19, 679-696 (2018).

27. Tong, M., Potter, J. A., Mor, G. \& Abrahams, V. M. Lipopolysaccharidestimulated human fetal membranes induce neutrophil activation and release of vital neutrophil extracellular traps. J. Immunol. 203, 500-510 (2019).

28. Khan, M. A. et al. JNK activation turns on LPS- and gram-negative bacteriainduced NADPH oxidase-dependent suicidal NETosis. Sci. Rep. 7, 3409 (2017).

29. Sollberger, G., Tilley, D. O. \& Zychlinsky, A. Neutrophil extracellular traps: the biology of chromatin externalization. Dev. Cell. 44, 542-553 (2018).

30. Azzouz, D. \& Palaniyar, N. ApoNETosis: discovery of a novel form of neutrophil death with concomitant apoptosis and NETosis. Cell Death Dis. 9, 839-841 (2018).

31. O’Neil, L. J. \& Kaplan, M. J. Neutrophils in rheumatoid arthritis: breaking immune tolerance and fueling disease. Trends Mol. Med. 25, 215-227 (2019).

32. Papayannopoulos, V., Metzler, K. D., Hakkim, A. \& Zychlinsky, A. Neutrophil elastase and myeloperoxidase regulate the formation of neutrophil extracellular traps. J. Cell. Bio. 191, 677-691 (2010).

33. Fonseca, Z. et al. Entamoeba histolytica induce signaling via Raf/MEK/ERK for neutrophil extracellular trap (NET) formation. Front. Cell Infect. Microbiol. https://doi.org/10.3389/fcimb.2018.00226 (2018).

34. Díaz-Godínez, C. et al. Entamoeba histolytica trophozoites induce a rapid non-classical NETosis mechanism independent of NOX2-derived reactive oxygen species and PAD4 activity. Front. Cell Infect. Microbiol. https://doi.org/ 10.3389/fcimb.2018.00184 (2018)

35. Nomura, K. et al. Citrullinated histone H3: early biomarker of neutrophil extracellular traps in septic liver damage. J. Surg. Res. 234, 132-138 (2019)

36. Thiam, H. R. et al. NETosis proceeds by cytoskeleton and endomembrane disassembly and PAD4-mediated chromatin decondensation and nuclear envelope rupture. Proc. Natl Acad. Sci. USA 17, 7326-7337 (2020).

37. Pao, K. C. et al. Activity-based E3 ligase profiling uncovers an E3 ligase with esterification activity. Nature 556, 381-385 (2018).

38. Swatek, K. N. \& Komander, D. Ubiquitin modifications. Cell Res. 26, 399-422 (2016).

39. Zheng, N. \& Shabek, N. Ubiquitin ligases: structure, function, and regulation. Annu. Rev. Biochem. 86, 129-157 (2017).

40. Buetow, L. \& Huang, D. T. Structural insights into the catalysis and regulation of E3 ubiquitin ligases. Nat. Rev. Mol. Cell Biol. 17, 626-642 (2016).

41. Rotin, D. \& Kumar, S. Physiological functions of the HECT family of ubiquitin ligases. Nat. Rev. Mol. Cell Biol. 10, 398-409 (2009).

42. Wang, Z. et al. A multi-lock inhibitory mechanism for fine-tuning enzyme activities of the HECT family E3 ligases. Nat. Commun. 10, 3162 (2019).

43. Mund, T. \& Pelham, H. R. Substrate clustering potently regulates the activity of WW-HECT domain-containing ubiquitin ligases. J. Biol. Chem. 293 , 5200-5209 (2018).

44. Casini, S. et al. Functional consequences of the SCN5A-p.Y1977N mutation within the PY ubiquitylation motif: discrepancy between HEK293 cells and transgenic mice. Int. J. Mol. Sci. https://doi.org/10.3390/ijms20205033 (2019).

45. Fang, J. Q. et al. TcpC inhibits toll-like receptor signaling pathway by serving as an E3 ubiquitin ligase that promotes degradation of myeloid differentiation factor 88. PLoS Pathog. 17, e1009481 (2021).

46. Hospenthal, M. K., Mevissen, T. E. T. \& Komander, D. Deubiquitinase-based analysis of ubiquitin chain architecture using Ubiquitin Chain Restriction (UbiCRest). Nat. Protoc. 10, 349-361 (2015).
47. Zhou, M., Li, S., Song, L., Hu, Q. \& Liu, W. 4-(2-(4-chlorophenyl)-1-((4chlorophenyl)amino)ethyl)benzene-1,3-diol is a potential agent for gout therapy as a dual inhibitor of XOD and NLRP3. Phytomedicine 42, 9-17 (2018).

48. Van Bemmelen, M. X. et al. Cardiac voltage-gated sodium channel Nav1.5 is regulated by Nedd4-2 mediated ubiquitination. Circ. Res. 95, 284-291 (2004).

49. Meyer-Schwesinger, $\mathrm{C}$. The ubiquitin-proteasome system in kidney physiology and disease. Nat. Rev. Nephrol. 15, 393-411 (2019).

50. $\mathrm{Li}, \mathrm{Y}$. et al. PSMD2 regulates breast cancer cell proliferation and cell cycle progression by modulating p 21 and p 27 proteasomal degradation. Cancer Lett. 430, 109-122 (2018).

51. Nijhawan, D. et al. Cancer vulnerabilities unveiled by genomic loss. Cell 150, 842-854 (2012).

52. Inoue, $\mathrm{M}$. et al. Plasma redox imbalance caused by albumin oxidation promotes lung-predominant NETosis and pulmonary cancer metastasis. Nat. Commun. 9, 5116 (2018).

53. Nguyen, G. T., Green, E. R. \& Mecsas, J. Neutrophils to the ROScue: mechanisms of NADPH oxidase activation and bacterial resistance. Front. Cell. Infect. Microbiol. 7, 373 (2017).

54. Gupta, S. \& Kaplan, M. J. The role of neutrophils and NETosis in autoimmune and renal diseases. Nat. Rev. Nephrol. 12, 402-413 (2016).

55. Wertz, I. E. \& Wang, X. From discovery to bedside: targeting the ubiquitin system. Cell Chem. Biol. 26, 156-177 (2018).

56. Hu, H. \& Sun, S. C. Ubiquitin signaling in immune responses. Cell Res. 26, 457-483 (2016).

57. Popovic, D., Vucic, D. \& Dikic, I. Ubiquitination in disease pathogenesis and treatment. Nat. Med. 20, 1242-1253 (2014)

58. Jiang, X. \& Chen, Z. J. The role of ubiquitylation in immune defence and pathogen evasion. Nat. Rev. Immunol. 12, 35-48 (2011).

59. Bhoj, V. G. \& Chen, Z. J. Ubiquitylation in innate and adaptive immunity. Nature 458, 430-437 (2009)

60. Liu, Y. et al. Dual ligand/receptor interactions activate urothelial defenses against uropathogenic E. coli. Sci. Rep. 5, 16234 (2015).

61. Metzler, KathleenD., Goosmann, C., Lubojemska, A., Zychlinsky, A. \& Papayannopoulos, V. A myeloperoxidase-containing complex regulates neutrophil elastase release and actin dynamics during NETosis. Cell Rep. 8, 883-896 (2014).

62. Loi, M., Raimondi, A., Morone, D. \& Molinari, M. ESCRT-III-driven piecemeal micro-ER-phagy remodels the ER during recovery from ER stress. Nat. Commun. 10, 5058 (2019).

63. Marchler-Bauer, A. et al. CDD: a Conserved Domain Database for the functional annotation of proteins. Nucleic Acids Res. 39, D225-D229 (2011).

\section{Acknowledgements}

We thank Professor Jianguo Xu (National Institute for Communicable Disease Control and Prevention, China) for providing the CFT073 strain of UPEC, and all blood donors. This work was supported by grants from the National Natural Science Foundation of China (81671613 to J.P.P.), the Postdoctoral Science Foundation of China (2020M671747 to J.Q.F), the Natural Science Foundation of Zhejiang Province (LQ20H100001 to J.Q.F.), and the Science and Technology Bureau of Zhejiang Province (LGD20H100001 to J.F.)

\section{Author contributions}

Q.O., J.Q.F., and J.P.P. contributed to the design of this study. Q.O., J.Q.F., Z.S.Z., Z.C. J.F., D.Y.X., K.Z.L., M.Q.Q, D.Y.Z, J.P.G., W.G., and N.R.Z. performed the experiments. Q.O., J.Q.F., Z.S.Z., and J.P.P analyzed the experimental data. J.P.P., Q.O., and J.Q.F. wrote the manuscript. Q.O. and J.Q.F. created Fig. 9. All authors reviewed and approved the final version of the manuscript.

\section{Competing interests}

The authors declare no competing interests.

\section{Additional information}

Supplementary information The online version contains supplementary material available at https://doi.org/10.1038/s41467-021-23881-8.

Correspondence and requests for materials should be addressed to J.-p.P.

Peer review information Nature Communications thanks the anonymous reviewer(s) for their contribution to the peer review of this work.

Reprints and permission information is available at http://www.nature.com/reprints

Publisher's note Springer Nature remains neutral with regard to jurisdictional claims in published maps and institutional affiliations. 
(c) (i) Open Access This article is licensed under a Creative Commons Attribution 4.0 International License, which permits use, sharing, adaptation, distribution and reproduction in any medium or format, as long as you give appropriate credit to the original author(s) and the source, provide a link to the Creative Commons license, and indicate if changes were made. The images or other third party material in this article are included in the article's Creative Commons license, unless indicated otherwise in a credit line to the material. If material is not included in the article's Creative Commons license and your intended use is not permitted by statutory regulation or exceeds the permitted use, you will need to obtain permission directly from the copyright holder. To view a copy of this license, visit http://creativecommons.org/ licenses/by/4.0/.

(C) The Author(s) 2021 